\title{
La decisión robótica: algoritmos, interpretación y justicia predictiva*
}

\section{Ettore Battelli*}

Resumen. A pesar de los riesgos de las decisiones robóticas, la previsibilidad y la justicia predictiva pueden ser consideradas como oportunidades para prever el resultado del juicio mediante el uso de algoritmos. El presente estudio nace a partir de una pregunta: ¿un juez puede ser reemplazado por un robot? La tesis propuesta es que la decisión robótica, así como la humana, puede contener errores y que las decisiones robóticas no podrán considerarse "justas", incluso cuando son lógicamente "correctas", ya que no existen máquinas infalibles. Sin embargo, se debería evaluar positivamente que la automatización del proceso decisorio permite lograr ventajas significativas en términos de uniformidad, fiabilidad y capacidad de control adecuado de la decisión robla de.

Palabras Clave: previsibilidad, justicia predictiva, algoritmos, decisiones robóticas, interpretación, seguridad jurídica.

\section{Robo-Decision: Algorithms, Legal Interpretation, Predictive Justice}

AbSTRACt. Predictability and predictive justice as an opportunity to foresee the judicial proceedings' outputs, considering all the risks related to the use of automated

* Fecha de recepción: 18 de marzo de 2020. Fecha de aceptación: 28 de agosto de 2020.

Para citar el artículo: BATTELLI, E., "La decisión robótica: algoritmos, interpretación y justicia predictiva", Revista de Derecho Privado, Universidad Externado de Colombia, n. ${ }^{\circ} 40$, enero-junio 2021, 45-86, DOI: https://doi.org/10.18601/01234366.n40.03.

** Università Roma Tre, Roma, Italia; profesor asociado. Doctor en Mercato e consumatori, Università Roma Tre, Roma, Italia. Contacto: ettore.battelli@uniroma3.it Orcid: 0000-0003-1387-3268. 
decision-makings. The study is based on a question: is the judge replaceable by a robot? The adopted approach aims at asserting that the robo-decisions and the humans' ones may be affected by misstatements and the automated decision-makings may not be regarded as "fair", even if they seem to be logically "correct": indeed, no algorithmic systems is infallible.

KEYWORDS: predictability, predictive justice, algorithms, robotic decisions, interpretation, legal certainty.

Sumario. I. Premisa metodológica y motivo de indagación. II. Primeras reflexiones y perspectivas de investigación en el contexto jurídico italiano. III. La aspiración a la objetividad y la despersonalización de la función jurisdiccional. IV. Datos, hechos y decisiones de la robot-justice. v. La justicia predictiva. vi. Experiencias y modelos probabilísticos en el marco de la justicia robótica. Conclusiones. Referencias.

\section{Premisa metodológica y motivo de indagación}

El presente estudio nace a raíz de una pregunta que no mucho tiempo atrás se relegaba a la ciencia ficción o, por decirlo así, a la ficción jurídica: ¿un juez puede ser reemplazado por un robot? Robots para los que, sea bien claro, el tipo penal de "homicidio" equivale, ante todo, como insinuaba la famosa película Una Odisea del espacio ${ }^{1}$, a la desactivación de la memoria central de un computador.

A nivel metodológico es necesario cuestionar primero si vale la pena perseguir un determinado objetivo, y solo si este resulta deseable se procedería a su estudio. Iniciemos identificando los motivos que podrían llevar a optar por este tipo de administración de justicia: (1) proporcionar rendimientos más eficientes que aquellos producidos por el ser humano, y (2) garantizar seguridad jurídica. Sobre este último es menester aclarar que, no obstante la certeza del derecho sea una aspiración básica en las comunidades humanas organizadas, en la ciencia jurídica también es patrimonio común la antigua enseñanza de Cicerón sintetizada en la expresión "summum ius, summa iniuria".

Efectivamente, aunque a nivel teórico es verdad que traducir el canon hermenéutico en una fórmula matemática debería garantizar un nivel más elevado de certeza en la interpretación de las normas y, por consiguiente, de previsibilidad de las decisiones procesales ${ }^{2}$, también es verdad que el famosísimo aforismo del más conocido jurista de la Antigua Roma nos recuerda que "la aplicación estricta de

1 Clarke, A. C., 2001. A Space Odissey, trad. ital. de B. Oddera, 2001. Odissea nello spazio, Milano, Longanesi, 1972, 175 ss.

2 Weber afirmaba que el derecho se basa en las reglas escritas y que la certeza del derecho no es más que la previsibilidad del resultado judicial. Cfr. WeBER, M., Economia e società, Milano, Bompiani, 1947. 
una norma puede convertirse en injusticia”. Así las cosas, preguntarnos si se puede reducir el derecho viviente -es decir, garantizar justicia en cada caso concreto- a la aplicación científica de un algoritmo parecería evidentemente nocivo, pero no es así.

Para brindar una respuesta a dicha pregunta, ante todo, es necesario interrogarnos sobre si el proceso de interpretación de las normas se puede traducir en un algoritmo ${ }^{3}$. Cabe resaltar que la expresión lingüístico-científica 'algoritmo' designa la capacidad de una máquina (propiamente un ordenador o un robot) de correlacionar grandes cantidades de información y de datos, todo ello según una fórmula determinada para finalmente dar el resultado previsto y requerido, así como ocurre en el cerebro humano.

En realidad, como es bien sabido, la sustitución tecnológica de los hombres con la inteligencia artificial (IA) va a influir en todos los procesos humanos en las próximas décadas, por lo tanto, es necesario profundizar en esta reflexión.

El primero de los dos motivos para incorporar la IA en el sector judicial, a saber, la exigencia de brindar prestaciones de "justicia" más eficientes, sin duda ha tenido una mayor difusión y se le considera prevalente.

En el ordenamiento italiano, por ejemplo, a lo largo de los últimos años se han llevado a cabo varias intervenciones en el ámbito del proceso civil, dirigidas a reducir los tiempos de la justicia y a volverla más eficiente: la reforma laboral (Ley del 11 de agosto de 1973, n. ${ }^{\circ}$ 533), el proceso sumario (Ley del 18 de junio de 2009, n. ${ }^{\circ}$ 69) y la mediación (Decreto legislativo del 4 de marzo de 2010, n. ${ }^{\circ} 28$ ), entre otras.

Sin embargo, en el plano de la actividad jurisdiccional está demostrado que la eficiencia, concebida como economicidad y rapidez, puede adoptar una fisionomía muy peculiar en comparación con otros sectores de la Administración Pública y con la economía.

En Italia, la llamada "deflación del contencioso" ha requerido a lo largo de los años un activismo por parte del legislador para introducir "barreras" procesales y procedimentales, el pago de una contribución (cada vez más gravosa) para los gastos relativos a la justicia, la previsión de límites a los pronunciamientos del juez y a los actos de los abogados. Todo ello con el fin de utilizar menos recursos, según las exigencias de la economicidad, pero también para hacer más rápidas las respuestas a la demanda de justicia de los ciudadanos. De hecho, rapidez y economicidad a menudo han ido de la mano. Lamentablemente, los efectos obtenidos se han limitado a hacer más difícil el papel del abogado y más onerosas para los ciudadanos las costas de acceso a la justicia.

Cabe también resaltar que la rapidez (objeto de varios pronunciamientos incluso de la jurisprudencia constitucional italiana) $)^{4}$, asumida como principio de la

3 Véase, para la noción de algoritmo, HofFmann-RiEm, W., "Verhaltenssteuerung durch Algorithmen - Eine Herausforderung für das Recht”, Archiv des öffent. Rechts, 142, 2017, 2 ss.

4 Luciani, M., "Garanzie ed efficienza nella tutela giurisdizionale”, Dir. soc., 2014, 433 ss. 
razonable duración del proceso, se ha introducido explícitamente en la Constitución italiana con el nuevo artículo 111.

En muchos casos, reducir los términos procesales de algunos pleitos conllevó ralentizar otros. "Si todo se acelera, nada se acelera, especialmente respecto de los recursos (humanos y materiales) no incrementados"5; sin olvidar que en ocasiones la rapidez va en detrimento de la calidad.

Una mayor rapidez podría ser útil solo si la prestación robótica pudiera garantizar el mismo estándar de calidad de la humana, asegurando el respeto del principio de tutela jurisdiccional efectiva. Solo de esta forma la decisión robótica podría, al mismo tiempo, garantizar la mayor seguridad jurídica arriba mencionada sin perder de vista los dos motivos que han originado esta reflexión ${ }^{6}$.

El punto de partida es una situación problemática: tiempos demasiado largos, falta de previsibilidad de las decisiones, recursos escasos, costes excesivos del sistema judicial en comparación con el resultado. A su vez, la ausencia de un sistema coherente y homogéneo genera conflictos. En este contexto la decisión robótica se considera una herramienta capaz de aportar mayor seguridad al tiempo que reduce la litigiosidad.

La tesis que se respalda es que la IA puede ser una herramienta útil para el sistema judicial moderno; una ayuda muy valiosa, si se usa bien, pero no en sustitución del juez.

No es casual que, por un lado, la informática haya sido incorporada a la justicia y que, por otro, se haya introducido un juicio civil casi totalmente digitalizado ${ }^{7}$ (aunque todavía no se haya extendido al juez de paz ni al Tribunal de Casación). En ese orden de ideas, traducir en lenguaje informático las reglas del proceso representa sin duda un objetivo importante.

Además, es apreciable una tendencia a la estandarización de los pronunciamientos de los jueces y de los actos de los abogados ${ }^{8}$, según un esquema estándar infor-

5 LuCIANi, M., "La decisione giudiziaria robotica", Riv. Associaz. Italiana Costituzionalisti, 2018, 3, 872 ss.

6 Ex multis para referir algunos términos mencionados Grossi, P., Ritorno al diritto, Roma-Bari, 2015, 51 ss.; LIPARI, N., "I civilisti e la certezza del diritto", Riv. dir. civ., 2015, 1115 ss.; IRTI, N., "Calcolabilità weberiana e crisi della fattispecie", Riv. dir. civ., 2014, 987 ss.; Rossi, P., "Razionalismo occidentale e calcolabilità giuridica", en CARLEO, A. (ed.), Calcolabilità giuridica, Bologna, Il Mulino, 2017, 32. Con respecto a las primeras aplicaciones de la IA en el ámbito judicial, cfr. CAstell, C. y Piana, D., "Giustizia predittiva. La qualità della giustizia in due tempi", Questione Giustizia, 4, 2018, 154 ss.

7 De Santis, A. D., "Processo telematico", Enc. Treccani Diritto-online, Roma, 2015; Poli, G. G., "Il sistema delle fonti del processo civile telematico", Riv. dir. proc., 2016, 4-5, 1201-1214.

8 El tema de la estandarización de los pronunciamientos ha adquirido cada vez más importancia en los últimos años con respecto a los diferentes perfiles frente a las exigencias de la IA. Se mencionan las circulares del CSM del 5/07/2017, del 22/11/2017 y del 20/06/2018, que realizan esquemas funcionales de pronunciamientos, identificados como modelos virtuosos. El CSM opera en virtud de una tendencia, también legislativa, que pretende el respeto del carácter breve tanto de la motivación como del recuento de los hechos y del desarrollo del proceso, para así acelerarlos, suponiendo que 
matizado que se propone eliminar la incertidumbre y el caos ${ }^{9}$. Todo ello conlleva beneficios en lo que se refiere a la claridad de las cuestiones y la rapidez de los procedimientos, optimiza la dialéctica entre las partes y valoriza la comunicación en el proceso, en el ámbito de un diálogo en el que los operadores del derecho, comenzando por los jueces, están llamados a conservar y custodiar el papel de sujetos con poder de decisión, a los que corresponde escoger entre las diversas opciones en materia de interpretación y del mérito de las respectivas cuestiones jurídicas ${ }^{10}$.

En especial, la estandardización de la motivación de la decisión es un primer paso hacia la posible "estandardización" de las decisiones tomadas por el juez que se apoya en el sistema informático ${ }^{11}$.

Naturalmente, la extraordinaria novedad de las modernas inteligencias artificiales es la posibilidad de procesar datos de forma masiva, agregándolos y disgregándolos entre sí, como solo un gran número de investigadores podría hacer, con una velocidad muy elevada que con dificultad podría ser alcanzada por un ser humano ${ }^{12}$.

Un ulterior paso que aquí ha sido considerado está representado por la capacidad por parte de la máquina de "transformar" los datos introducidos, interpretándolos y activando un razonamiento lógico típico de la mente humana, en actos o contratos ${ }^{13}$.

En todo caso, no obstante la capacidad de las máquinas de aprender de forma autónoma a través del llamado "machine learning", o incluso de procesar nuevas rutas de aprendizaje con el llamado “deep learning”, les falta la capacidad, totalmente humana, de evaluar las abundantes variables imprevistas o imprevisibles: el llamado discernimiento ${ }^{14}$.

la concisión y la brevedad aseguren que se libera tiempo al juez para que pueda usarlo en ulteriores actividades del despacho. La intervención del CSM se propone como herramienta para limitar la libertad en la redacción de las sentencias tanto penales como civiles, no solo en relación con el dato cuantitativo de la extensión, sino también para restablecer a la motivación en una función esencial de argumentación jurídica, que además debe recaer sobre los hechos relevantes a efectos de la decisión.

9 Belloso Martin, N., "Algoritmos predictivos al servicio de la justicia: una forma de minimizer el riesgo y la incertidumbre", Revista da Faculdade Mineira de Direito, Puc Minas / Dossiê - Jorge Eduardo Douglas Price e Raffaele De Giorgi, vol. 22, n. ${ }^{\circ}$ 43, 2019, 1-31.

10 Irti, N. y Severino, E., "Le domande del giurista e le riposte del filosofo (un dialogo tra diritto e tecnica)", Contratto e Impresa, 2006, 665 ss.; SEVERINo, E., La potenza dell'errare, Milano, Rizzoli, 2013, 87 ss.

11 BıснI, R., "Intelligenza artificiale e diritto - intelligenza artificiale tra 'calcolabilità' del diritto e tutela dei diritti", Giur. it., 2019, 7, 1773.

12 CRISCI, S., "Intelligenza artificiale ed etica dell'algoritmo", Foro Amministrativo, 10, 1787.

13 Di SABATo, D., "Gli smart contracts: robot che gestiscono il rischio contrattuale”, Contr. Impr., 2017, 378.

14 Cuando se habla de "machine learning" se hace referencia a los varios mecanismos que permiten a una máquina inteligente mejorar las propias capacidades y rendimientos en el tiempo. La máquina, por lo tanto, será capaz de cumplir tareas específicas mejorando, mediante la experiencia, sus capacidades, respuestas y funciones. Distinto es el concepto de Deep Learning, que es un sistema de aprendizaje que, utilizando las estructuras de redes neurales, elabora grandes sets de datos y conduce a un resultado semejante al que podría alcanzar el hombre. A través de este sistema la máquina aprende con el ejemplo. 


\section{Primeras reflexiones y perspectivas de investigación en el contexto jurídico italiano}

La capacidad de procesar enormes cantidades de datos y conocimientos ha permitido la evolución de las herramientas de IA, capaces de utilizar, manipular y combinar mejor los big data ${ }^{15}$, que hoy en día representan la nueva frontera de aplicación de la IA, incluso en el ámbito del derecho ${ }^{16}$.

Además, no hay que subestimar las potencialidades de la formación de la base de datos de las decisiones a nivel nacional, proyecto en el cual está trabajando el CNF (Consejo Nacional de Abogados en Italia), partiendo del presupuesto de que la máxima disponibilidad de los datos es imprescindible para garantizar la uniformidad del derecho.

En la prospectiva de automatizar la decisión, existen en Italia muchas experiencias de ODR -online dispute resolution- destinadas a la creación de una nueva relación juez-partes y a la maximización del objetivo de adelantar conciliaciones, en las que la composición de los intereses se realiza por medio de algoritmos creados para la asignación de bienes más que de derechos.

La tecnología, por lo tanto, ha entrado plenamente en el amplio mundo de la justicia y ha demostrado resultados significativos, como contribuir a reducir las decisiones judiciales represadas en la jurisdicción civil italiana, en comparación con tiempos no lejanos.

Manteniéndonos en el marco de los dos motivos de investigación mencionados, en lo que se refiere a la rapidez de los procesos, inclinar la balanza hacia una decisión robótica resulta necesariamente condicionado, en el sentido de que es necesario constatar que la prestación robótica y aquella intelectual humana ${ }^{17}$ sean equivalentes a nivel cualitativo, sin perjudicar el principio de la tutela jurisdiccional plena y efectiva del ciudadano y de la empresa.

En lo que se refiere a los costes, no es seguro que los gastos relativos a la proyección, realización y mantenimiento de una estructura robótica se revelen menos dispendiosos que los del ordinario sistema judicial operado por seres humanos. Sería necesario realizar cálculos rigurosos antes de poder afirmar con seguridad que, desde

15 KAPLAN, J., Intelligenza artificiale. Guida al futuro prossimo, LuIss University Press, Roma, 2017, 52 ss.

16 SARToR, G., Intelligenza artificiale e diritto. Un'introduzione, Milano, Giuffrè, 1996, 19 ss.

17 Las "tres D" del debate anglosajón (dull, duty, dangerous) es el trabajo del cual el hombre espera que el robot lo libre. La cuestión que se plantea es un trabajo que tiene por objeto rendimientos "intelectuales", fruto de satisfacción y motivo de realización en la vida del hombre. Véase Lin, P., ABNEY, K. y BeKEy, G., "Robot ethics: Mapping the issues for a mechanized world", Artificial Intelligence 175, 2011,944. Basta recordar que Isaac Asimov, que en general fue bastante acertado en sus previsiones, fracasó en Io, Robot (Bompiani, Milano, 1985, 37) mientras imaginaba que la producción de robot a robot habría sido impedida por los sindicatos. 
el punto de vista económico, la opción robótica sea más conveniente que la opción humana ${ }^{18}$.

Es verdad que las decisiones robóticas pueden aportar importantes beneficios -no solo por su intrínseca capacidad para la racionalización del proceso de decisión, con elevados niveles de rapidez y eficiencia y con herramientas para la reducción de las desigualdades a través de la lucha contra los fraudes ${ }^{19}$, , pero también es verdad que, a partir de las evidencias empíricas existentes, las decisiones robóticas suelen "codificar" el pasado, capturando soluciones y predicciones en esquemas procedentes de acontecimientos históricos y de valores que han dirigido la programación del algoritmo en que se apoya el proceso decisorio ${ }^{20}$.

Por esta razón es necesario reducir al mínimo los riesgos de impacto negativo en los derechos civiles, sociales y políticos de las personas ${ }^{21}$. En esta perspectiva, es normal dudar tanto de la utilidad del desarrollo de algoritmos específicos de aprendizaje autónomo para encontrar y corregir la existencia de sesgos en la toma de decisiones (fairness formulas) ${ }^{22}$ como de la adopción de códigos de autorregulación de los sujetos implicados ${ }^{23}$. Dichas herramientas son útiles sin duda, pero se mueven en una lógica de autodisciplina que, por su propia naturaleza, está supeditada solo a aquellos vínculos que la cultura de los operadores pueda sugerir en un determinado momento histórico ${ }^{24}$.

Volviendo a la exigencia objetiva de certeza, con su "perfil de confiabilidad"25 por parte de los ciudadanos, tan relevante en los complejos ordenamientos contemporáneos, necesariamente debemos convenir en que una justicia "previsible" es de gran interés para los empresarios o, en general, para aquellas personas que quieren invertir su capital. Sin embargo, un derecho "calculable"26 -concebido como fundamento absoluto de seguridad jurídica, más aún en la actual realidad normativa

18 Por todos, cfr. Douglas Price, J. E., La decisión judicial, Buenos Aires, Culzoni, 2012.

19 Resta, G., "Governare l'innovazione tecnologica: decisioni algoritmiche, diritti digitali e principio di uguaglianza", Pol. diritto, 2, 2019, 199 ss., esp. 218.

20 Véase O’NeIL, C., Weapons of Math Destruction: How Big Data Increases Inequality and Threatens Democracy, London, Penguin, 2016.

21 Cardon, D., "Le pouvoir des algorithmes", Pouvoirs, 164, 1, 2018, 63-79.

22 Desai, D. R. y Kroll, J. A., "Trust but verify: a guide to algorithms and the law", Harvard J. Law \& Tech., 1, 2018, 35 ss.

23 Borgesius, F. Z. (ed.), Discrimination, Artificial Intelligence, and Algorithmic Decision-Making, Strasbourg, Council of Europe, 2018, 10 ss., esp. 27.

24 Esto implicaría descartar tanto un modelo desordenado de "capitalismo de la seguridad", que terminaría por anclar a la sociedad a sus iniquidades y prejuicios, codificándolos en el lenguaje informático, como un sistema programado de "vigilancia del Estado" (BARocas, S. y SElbst A.D., "Big data's disparate impact", Cal.L.Rev., 2016, 104,671 ss.).

25 Costantino, G., "Il principio di affidamento tra fluidità delle regole e certezza del diritto", ponencia en el evento "Norme processuali e mutamenti di giurisprudenza" (Roma, 24 de mayo de 2011), Riv. dir. proc., 2011, 5, 1073-1097.

26 IRTI, N., "Per un dialogo sulla calcolabilità giuridica", en AA.vv., Calcolabilità giuridica, cit., 21 ss. 
compleja y globalizada- representa simplemente una aspiración -o poco más que eso-, que además no es nueva para la humanidad ya que esta la persigue desde hace al menos un par de siglos (por ejemplo con las codificaciones de principios del siglo $\mathrm{XIX}$ ), o incluso dos milenios (si se consideran las monumentales obras de reorganización del derecho romano).

Entre múltiples autores cabe traer a colación a Leibniz, que ya en 1666 se proponía extender al dominio de la jurisdicción el ideal de la calculabilidad universal, que basaba su reflexión en la lógica. Él afirmaba que era necesario reducir el lenguaje a la simplicidad, para así poder calcular errores y razones (la llamada ars combinatoria), y expresaba su deseo de que "Las partes un día, frente a una disputa, podrán sentarse y realizar un cálculo" 27 , suponiendo así una calculabilidad que conllevaba plena previsibilidad: "Todas las cuestiones de derecho puro pueden definirse con certidumbre geométrica" 28 .

Partiendo del hecho de que distinguir entre robot e IA es difícil ${ }^{29}$, normalmente cuando hablamos de 'robot' nos referimos a la idea de "un conjunto de metal y plástico" que parece un ser humano o que es capaz de replicar las acciones humanas $^{30}$, mientras que con 'inteligencia artificial' 31 se hace referencia a la capacidad

27 LeIBNIZ, G. W., Dissertatio de arte combinatoria, 1666.

28 Leibniz, G. W., "Princìpi ed esempi della scienza generale", en Barone, F. (ed.), Scritti di logica, Milano, Mondadori, 2009, 121; cabe recordar que el mismo pensador se imaginaba también una lengua universal capaz de abarcar todos los conocimientos de la humanidad, concibiéndola como una lengua específicamente "numérica"; precursor en lo que atañe al lenguaje "binario" ideado por Alan Turing para instruir las máquinas "inteligentes". Véase TuRING, A., Proposal for the Development in the Mathematics Division of an Automatic Computing Engine (ACE). Part I: Descriptive Account, informe presentado al Executive Committee del National Physics Laboratory, 1945, en AA.vv., Collected Works of A.M. Turing, Mechanical Intelligence, Ámsterdam, Elsevier, 1992, trad. ital. de G. Lolli y N. Dazzi, Proposta per lo sviluppo nella Divisione Matematica di una macchina calcolatrice elettronica (ACE). Parte I: Presentazione descrittiva, ahora en Intelligenza meccanica, Torino, Bollati Boringhieri, 1994, 35. Antes de que se aplicara a las máquinas, es necesario recordar que la elaboración del código binario es atribuida a Nepero por KAPLAN, R., The Nothing that is. A Natural History of Zero, trad. ital. de S. Galli y C. Capararo, Zero. Storia di una cifra, Milano, RCs, 2014, 297 ss.

29 Véase Balkin, J. M., "The Path of Robotics Law”, en Calif. L. Rev., 2015, 45.

30 Citando Holder, C., Khurana, V., Harrison, F. y Jacobs, L., "Robotics and law: key legal and regulatory implications of the robotic age", I, Computer Law and Security Review, 2016, 384: "A machine resembling a human being and being able to replicate certain human movements and functions automatically". No son pocas las propuestas, entre ellas la que figura en Lin, P., ABNEY, K. y BeKey, G., Robot Ethics, MIT Press, 2012, 943: "an engineered machine that senses, thinks and acts".

31 García-Prieto Cuesta, J., “QQué es un robot?”, en Barrio Andrés, M. (dir.), Derecho de los robots, Madrid, La Ley, 2018, 46: "Comúnmente también se considera que existe inteligencia artificial cuando una máquina imita funciones cognitivas de organismos vivos". SAuvé, J.-M., Le juge administratif et l'intelligence artificielle, Conférence des présidents des jurisdictions administratives, Rennes, viernes 20 de abril de 2018, 1, menciona que el concepto de "inteligencia artificial" fue introducido en 1955 por dos informáticos americanos, John McCarthy y Claude Shannon, durante una conferencia organizada por los mismos sobre la "inteligencia artificial" que tuvo lugar en 1956. 
de la "máquina" de interactuar con el exterior "imitando" los procesos mentales humanos ${ }^{32}$.

Entonces, si lo que se pide a la máquina no es que realice lo que un sujeto ha decidido, sino que decida de forma autónoma, nos encontramos frente a una mutación tanto de la máquina como de la tecnología en la que se basa, que de "herramienta" pasa a ser "sujeto". Por esta razón se habla cada vez más de agentes humanos como sistemas tecnológicos automatizados proyectados para tomar decisiones o expresar evaluaciones por su propia cuenta ${ }^{33}$.

En este contexto es imprescindible identificar las pretensiones de iuris-dictio (id est: ejercer la función judicial, concepto diferente al de garantizar "justicia") tradicionalmente dirigidas a un juez-humano y que sería deseable que se exigieran a los robots con resultados al menos equivalentes a lo que legítimamente se podría pretender del primero ${ }^{34}$.

Por consiguiente, del juez robot no hay que pretender, legítimamente, la decisión "justa", sino la decisión "exacta", es decir, que aplique de forma correcta las fuentes pertinentes ${ }^{35}$, posiblemente al final de un proceso "justo", entendiendo con esto que respete los procedimientos previstos según el principio de legalidad.

\section{La aspiración a la objetividad y la despersonalización de la función jurisdiccional}

Si lo que acabamos de expresar resulta claro, existe una tercera razón que, más que las analizadas hasta ahora, podría ser argüida a favor de una justicia robótica, esto es, la "exactitud" de una decisión "objetiva", considerando que si un robot es capaz de realizar operaciones matemáticas extremamente complejas con una rapidez y una exactitud de manera mucho más precisa, neutra y rápida que los seres humanos,

32 Véase Neumann, J. von, The Computer \& the Brain, New Haven, Yale University Press, 1958, trad. ital. de P. Bartesaghi, Computer e cervello, Milano, Il Saggiatore, 2014, 96, 115.

33 Simoncini, A., "L'algoritmo incostituzionale: intelligenza artificiale e il futuro delle libertà", BioLaw Journal - Rivista di BioDiritto, 2019, 1,63 s., que invita a reflexionar también sobre las formas de decisión indirectas, en las cuales los "agentes humanos" asumen decisiones, aun sin la necesaria conciencia, basándose en medidas cada vez mayores sobre informaciones proporcionadas por sistemas tecnológicos (más o menos) automatizados. Por ejemplo: los GPs, la gestión del tráfico aéreo, los autos sin conductor, la domótica e incluso la publicidad en la web. De hecho, sucede que las decisiones (desde el clima a la salud, desde los programas de cine a los deportes, a la ruta más corta para llegar a un lugar, etc.) están prácticamente basadas en conocimientos transmitidos por plataformas tecnológicas (como los motores de búsqueda y las redes sociales), utilizables a través de una serie de objetos de uso común (el ordenador y también el smartphone) (p. 65). Esto nos lleva a concluir que el grado de dependencia de las decisiones humanas respecto de la tecnologia, y la asimetría entre las partes y las llamadas High Tech, implican que la misma libertad del consentimiento sea una mera fictio. Es así que, por un lado, si el sujeto no da su consentimiento al "registro" de los datos no tiene acceso a servicios que, por otro lado, en su mayoría son básicamente gratuitos.

34 Luciani, M., La decisione giudiziaria robotica, cit., 878.

35 Véase Gentili, A., Senso e consenso., vol. I, Storia e teoria, Torino, Giappichelli, 2015, 202 ss., con amplia bibliografía. 
se espera que también esté a la altura de la tarea en caso de tener que proporcionar prestaciones judiciales ${ }^{36}$.

Dicha exigencia se coloca perfectamente en continuidad con la tendencia, que surgió entre finales del siglo XVIII y finales del siglo xix y que duró más de un siglo, a una progresiva despersonalización de la figura del juez, cuya actividad resulta cualificada cada vez menos por sus virtudes individuales y mucho más por su profesionalidad; esto es, no por su sabiduría sino por su saber, no por sus dotes morales sino por las intelectuales. De hecho, hemos sido testigos de la transición del modelo de referencia, cuyos orígenes son muy antiguos, del juez-oráculo al juez-funcionario.

Como es notorio, se subsiguieron sensibilidades jurídico-filosóficas muy diferentes entre ellas (el realismo escandinavo, el americano ${ }^{37}$, la hermenéutica contemporánea, la doctrina de la pre-comprensión, el modelo analítico), pero a todas las acomunaba la tendencia a afirmar la insignificancia del texto de la disposición legislativa y a brindar atención a la "realidad" de la norma "creada" por el juez. No obstante, precisamente la automatización de la jurisdicción confiada al robot podría incluso acercar los dos "mundos distintos" (descritos por Kelsen ${ }^{38}$ ): el de la naturaleza, gobernado por el principio de causalidad, y el de la cultura, gobernado por normas sociales.

Para que esto pudiera ocurrir sería necesario pronosticar una exactitud matemática y la unidad del derecho para realizar, al menos de manera abstracta, una justicia mecánica delegada a los jueces robot. Sobre la base de la evolución sumariamente delineada, la justicia robótica parece, en el mejor de los casos, un "paradigma idealtípico" que nace como reacción ante aquellas doctrinas de la interpretación que a menudo acabaron legitimando la arbitrariedad y el subjetivismo del juez.

Mientras tanto, aumentan las dudas sobre la factibilidad tout court de una robotjustice capaz de prescindir del factor humano, ya que renunciar al componente humano parece hoy en día una hipótesis remota ${ }^{39}$.

Más allá del debate aquí mencionado -que en el ordenamiento italiano puede conectarse con la "deseada" interpretación uniforme de la ley, relativa al artículo 65 del Decreto real 12 de 1941 sobre el ordenamiento judicial-subsisten fuertes dudas sobre la posibilidad de transformar cada decisión en modelos matemáticos, caracterizados por el automatismo de los algoritmos ${ }^{40}$.

36 Véase Luciani, M., La decisione giudiziaria robotica, cit., 880.

37 AlPa, G., "Note sulla calcolabilità nel diritto nordamericano", en AA.vV., Calcolabilità giuridica, cit., 88 , donde se destaca la base filosófica en que se basan los primeros casos de justicia predictiva (en particular la corriente del realismo jurídico americano).

38 Kelsen, H., Society and Nature. A Sociological Inquiry, Chicago (Ill.), The Univ. of Chicago Press, 1943, trad. ital., Società e natura. Ricerca sociologica, Torino, Einaudi, 1953, 18 ss.

39 LuCIANI, M., La decisione giudiziaria robotica, cit., $881 \mathrm{s.}$

40 En 2004, el Presidente Agregado honorario del Tribunal Supremo y docente de informática jurídica, Borruso, R., L'informatica nel diritto, Milano, Giuffrè, 2004, 316 s. afirmó que la ley puede ser informatizada y construida mediante algoritmos. Al respecto, cfr. Viola, L., Interpretazione della legge con modelli matematici. Processo, a.d.r., giustizia predittiva, Milano, Diritto Avanzato, 2018, 2. ${ }^{\mathrm{a}}$ ed., 33 . 
Por otra parte, el mismo legislador, por ejemplo, sin querer vuelve inutilizable todo tipo de modelo matemático posible como demuestra, en el proceso declarativo sumario, el inciso que consagra que el juez "actuará de la forma que considere más adecuada" (art. 702 ter inc. 5 del código de procedimiento civil, c.p.c., italiano); a su vez, es un ejemplo el campo del derecho de familia. De hecho, cuando la ley le atribuye tan amplios poderes al juez, se precluye el empleo de modelos matemáticos. Por consiguiente, así como es cierto que la interpretación tiene que responder a exigencias de objetividad, también es importante que dicha interpretación tenga que considerar sus límites, establecidos por el mismo legislador que, en algunos casos, atribuye mayor discrecionalidad al intérprete ${ }^{41}$.

El Tribunal Constitucional italiano, por su parte, ha sostenido en diversas ocasiones que el juez no puede ser vinculado a presunciones absolutas, es decir, a automatismos respecto de la decisión ${ }^{42}$. En estos casos, y no solamente en el derecho penal, el juez no puede estar totalmente "vinculado" a ningún automatismo que le impida "personalizar" la decisión incluso yendo más allá de los esquemas pre-determinados ${ }^{43}$. Siguiendo esta misma línea lógica, en efecto, encontramos también la jurisprudencia administrativa ${ }^{44}$, que rechaza todo tipo de hipótesis de uso exclusivo de algoritmos en las decisiones administrativas.

Así pues, al menos en Italia, aún predomina la idea de que una decisión jurídicamente relevante que se base en un algoritmo, aunque el mismo esté bien estructurado en el marco de la predicción, resulta de todas formas en contraste con la teoría jurídica misma si su uso implica que no se puedan proporcionar razones lógicas comprensibles $^{45}$, ya que estas serían interpretables solo por parte de los matemáticos ${ }^{46}$. La decisión de los robots garantiza sin duda un riguroso alineamiento al proceso de

41 Viola, L., "L’interpretazione della legge ex art. 12 Preleggi si basa su un algoritmo", Giustiziacivile com, 22.02.2019, el cual afirma que la decisión robótica, en la legislación italiana, para ser claros en el razonamiento que se quiere hacer, no podría dejar de tener en cuenta como referente principal el artículo 12 de las llamadas "Preleyes" del código civil, que certifica lo siguiente: "Al aplicar la ley no se le puede atribuir otro sentido que el evidenciado por el propio significado de las palabras según la conexión de las mismas, y por la intención del legislador. - Si una controversia no puede ser decidida con una norma precisa, se tendrán en cuenta las normas que regulan casos similares o materias semejantes; si subsisten dudas sobre el caso, se decidirá según los principios generales del ordenamiento jurídico del Estado".

42 Ex multis, Corte Cost., n. 139/1982 y n. 367/2004.

43 La cuestión es que los automatismos gozan de una plusvalía "práctica” relacionada con las decisiones sugeridas automáticamente (por el sistema, por el algoritmo, por la elaboración de perfiles automáticos), que podemos no aplicar, a condición de que se quiera asumir un considerable esfuerzo (y riesgo) de valoración. Pensemos, por ejemplo, en los riesgos que puede correr quien decide ignorar las valoraciones automáticas producidas por máquinas en el sector del diagnóstico médico.

44 Véase Patroni GrifFi, F., "Tecniche di decisione e prevedibilità nella sentenza amministrativa”, en AA.vv., Calcolabilità giuridica, cit., $183 \mathrm{~s}$.

45 Viola, F., Zaccaria G. y Pastore, B., Le ragioni del diritto, Bologna, Il Mulino, 2017.

46 Cfr., por todos, Gadamer, H. G., Wahrheit und Methode, Tübingen 1960, 1965, 2. a ed., trad. ital., Verità e metodo, G. Vattimo (trad.), Milano, Bompiani, 1972, 1990; ZACCARIA, G., Ermeneutica e giurisprudenza. I fondamenti filosofici nella teoria di Hans Georg Gadamer, Milano, Giuffrè, 1984 
decisión, ya que este se basa en un algoritmo capaz de garantizar un mayor grado de exactitud. La máquina puede, por tanto, garantizar objetividad solo dentro de los límites de la precisión del proceso de decisión ${ }^{47}$, mientras que el resultado de la decisión sigue siendo subjetivo y estrictamente relacionado con los datos y los hechos del respectivo proceso ${ }^{48-49}$.

\section{Datos, hechos y decisiones de la robot-justice $e^{50}$}

A pesar de la crisis del trinomio hecho-norma-efecto (en italiano, la "crisi della fattispecie") $)^{51}$, la parte preponderante de la actividad del juez sigue siendo la subsunción del hecho en la norma o, dicho de otra manera, conectar la circunstancia concreta a la circunstancia abstracta. En ese orden de ideas, adquieren una importancia decisiva los hechos "comunicados" al juez (humano) y que representan el inevitable resultado de una selección de hechos relativos a la res iudicanda. Si el juez humano fuera reemplazado por el juez robot, los datos "digitalizados" introducidos en la "máquina pensante" no incluirían todos los datos disponibles, ya que se trataría de todas formas de los que se hayan considerado relevantes y que son accesibles en la dimensión que se suele denominar "datasphere" 52 .

La única diferencia entre el juez con inteligencia humana y el robótico dotado de IA sería, por tanto, que el primero adquiere los datos sobre la base de los hechos que se le han "representado", mientras que respecto del juez robot dichos datos se "introducen"; de esta manera, en ambos casos la "exactitud" de la decisión estará condicionada por la totalidad y la pertinencia de los datos que los terceros habrán proporcionado al juez, sea humano o sea un robot $^{53}$.

Sin embargo, en uno y otro caso, si entre los infinitos datos y hechos se selecciona solamente una parte, entonces la exactitud de los cálculos útiles para la sub-

47 Desde una perspectiva eminentemente procesal, cfr. NIEva Fenoll, J., Inteligencia artificial y proceso judicial, Madrid, Marcial Pons, 2018.

48 Belloso Martin, N., Algoritmos predictivos al servicio de la justicia, cit., 9: "La inteligencia artificial trabaja con algoritmos cuya comprensión no está al alcance de los ciudadanos, por lo que se puede llegar a lo que se ha denominado como la 'dictadura del algoritmo', entendiendo por tal algoritmo una secuencia de instrucciones secuenciales mediante las cuales pueden llevarse a cabo ciertos procesos y darse respuesta a determinadas necesidades o decisiones".

49 Mattera, R., "Decisione negoziale e giudiziale: quale spazio per la robotica?", Nuova giur. civ. comm., 2019, 201.

50 Katsh E. y Rabinovich-Einy, O., Digital Justice. Technology and the Internet of Disputes, Oxford, Oxford University Press, 2017.

51 IRTi, N., "La crisi della fattispecie", en ID., Un diritto incalcolabile, Torino, Giappichelli, 2016, 19 ss.

52 Bergé, J.-S., Grumbach, S. y Zeno-Zencovich V., "The 'Datasphere'. Data flows beyond control, and the challenges for law and governance", Eur. J. Comp. Law \& Gov., 5, 2, 2018, 144-178.

53 Luciani, M., La decisione giudiziaria robotica, cit., 883. 
sunción del hecho a la norma sigue igualmente dependiendo de un proceso cognitivo humano y, como tal, "falible" 54 .

Por consiguiente, la decisión robótica, al igual que la humana, estará sujeta a errores y las decisiones robóticas no podrán considerarse exactas ya que no existen máquinas infalibles ${ }^{55}$, no porque arrojen resultados basados en una lógica imperfecta de instrucciones proporcionadas a la máquina, sino por una introducción defectuosa o incompleta de datos erróneamente considerados relevantes ${ }^{56}$. Hay que tener en cuenta que cualquier máquina puede equivocarse.

La cuestión es diferente cuando el robot se presenta como alter ego del juez, ya que el primero ayuda al segundo a tomar conciencia de la manera en que está razonando y decidiendo, apoyándolo para una correcta toma de decisiones, con el fin de integrar, y no de reemplazar, su actuar lógico-jurídico ${ }^{57}$.

Si lo mencionado se considera aceptable, se podría entonces convenir en que la inmediata consecuencia sería que no se podría hablar de la seguridad del derecho y, en su lugar, lo que sería seguro es la falibilidad de la máquina que tuviera que desempeñar las funciones del juez en lugar del hombre.

Ciertamente se podría objetar que el juez robot resultaría de todas formas, desde diversos puntos de vista, más fiable que el ser humano, por lo que la solución de la jurisdicción robótica, aunque no perfecta, sería preferible ${ }^{58}$.

Si la decisión resultado de la IA depende no solo de lo que se ha descrito arriba sino también del algoritmo utilizado, que "consiste en una secuencia de instrucciones con la que el calculador elabora un proceso de cálculo"59 , la decisión será entonces el resultado derivado del programador que creó el código de procesamiento de datos y de la mente que seleccionó los datos introducidos ${ }^{60}$.

54 Zellin, P., La dittatura del calcolo, Milano, Adelphi, 2018, 136.

55 Véase Belloso Martin, N., Algoritmos predictivos al servicio de la justicia, cit., 16: "dictadura del algoritmo vs. decisión judicial".

56 Ya Turing, A. M., Proposal for the Development, cit., 57, identificaba tres posibles factores de error: fallos de instalación, interferencias temporales, tablas de enseñanza incorrectas. Luego NeumAnN, J. von, Computer e cervello, cit., 82, advertía que en los procedimientos de cálculo complejos los errores pueden superponerse y multiplicarse.

57 Punzi, A., L'ordine giuridico delle macchine. La Mettrie - Helvétius - D'Holbach.L'uomo macchina verso l'intelligenza collettiva, Torino, Giappichelli, 2003.

58 Véase IASELli, M., "È ipotizzabile l'avvocato-robot o il giudice-robot? Le applicazioni dell'intelligenza artificiale nel settore legale", Altalex.com, 15 de junio, 2017.

59 Véase Zeluin, P., La dittatura del calcolo, cit., 11 y Ferragina, P. y Luccio F., Il pensiero computazionale. Dagli algoritmi al coding, Bologna, Il Mulino, 2017, 8 ss., donde se destaca que el algoritmo "establece las reglas lógicas a utilizar en la resolución de un problema y la secuencia según la cual tienen que ser aplicadas", y se diferencia del coding, que "ordena la ejecución de los pasos de aquella secuencia sobre un dispositivo específico de cálculo". Neumann, J. von, Computer e cervello, cit., 127, había definido el código como "un sistema de instrucciones lógicas que un robot sea capaz de realizar y que le permita llevar a cabo una cierta operación organizada".

60 Véase Avitabile, L., "Il diritto davanti all'algoritmo", Riv. it. sc. giur., 2017, 319 ss. 
Si trasladamos la decisión judicial del hombre al robot, esencialmente se traslada el poder de un hombre a otro hombre, no de un hombre a una máquina. Todo ello hasta cuando tengamos máquinas totalmente autónomas y que puedan reproducirse de forma autónoma ${ }^{61}$.

Si la subsunción de la circunstancia concreta en la circunstancia abstracta es una actividad silogística, no implica que también lo sea la reconstrucción de la circunstancia abstracta a través de la interpretación por parte de un juez, dotado de IA, aunque en realidad sería posible introducirle instrucciones en ese sentido ${ }^{62}$. De hecho, se trataría, incluso en este caso, de "enseñarle" al robot el uso de los criterios interpretativos que los juristas, dotados de la simple y falaz inteligencia humana, suelen utilizar ${ }^{63}$.

La interpretación mecánica del juez robot sería simplemente una interpretación matemática basada en datos y algoritmos elaborados por medio de una máquina ${ }^{64}$. Pero hay que considerar que, por muy claras que fueran las palabras consignadas en una ley, siempre es necesario relacionar las palabras del texto con el contexto ${ }^{65}$, siempre es útil conocer los antecedentes, siempre es importante tener en cuenta la realidad social del tiempo en el que estas palabras tienen que ser aplicadas, siempre hay que considerar la ratio de la norma. Así, por ejemplo, puede que diversas normas no se apliquen según un criterio estrictamente literal, porque el resultado sería absurdo o injusto. Dichos resultados se pueden evitar empleando precisamente los criterios de interpretación o criterios hermenéuticos, ya que -no hay que olvidarlo-

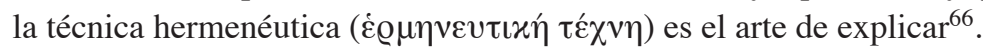

\section{La justicia predictiva}

El recorrido de la toma de decisiones con la intervención de la IA se configura ante todo creando bases de datos que contengan el conjunto de normas; el archivo de

61 El razonamiento es similar a la hipótesis de la denominada centralización de la responsabilidad del productor del vehículo planteada por Holder, C. et al., Robotics and Law, cit., 386. Como ilustró B. Romano en Dalla metropoli verso internet. Persona. Libertà. Diritto, Torino, Giappichelli, 2017, 112, lo importante es la acción humana basada en el "razonamiento" robótico. Además, la mayor atención de los reguladores se está concentrando en el tema de la responsabilidad; véase, p. ej., la Resolución del Parlamento Europeo P8_TA (2017)0051 del 16 de febrero de 2017.

62 Luciani, M., La decisione giudiziaria robotica, cit., 887.

63 Viola, L., L'interpretazione della legge ex art.12..., cit., 2019.

64 Oliva Santos, A. De la, “'Giustizia predittiva', interpretazione matematica delle norme, sentenze robotiche e la vecchia storia del 'Justizklavier'”, Riv. trim. dir. proc. civ., 3, 2019, 883 ss. esp. 887.

65 Gentili, A., Senso e consenso, i, cit., 180 ss.

66 De este modo Oliva SAnTos, A. De LA, "Giustizia predittiva", cit., 888, muy crítico con la robotjustice. Es menester aclarar que "Jurimetría" es el nombre de la plataforma de justicia predictiva elaborada por la multinacional Wolters Kluver y propuesta en España. Se menciona, en este contexto, la ponencia de Oliva SANTOS, A. DE LA, "Informática, interpretación (¿inexistente por innecesaria o matemática?) De la ley y la vieja historia del 'Justizklavier'", realizada en la Conferencia internacional de estudios "Giustizia predittiva e prevedibilità delle decisioni. Dalla certezza del diritto alla certezza dell'algoritmo?”, Università degli studi di Bari Aldo Moro, 5 de octubre de 2018. 
la jurisprudencia sobre los precedentes; la predisposición de modelos de redacción judiciales; el uso de un lenguaje jurídico predeterminado y vinculado a nivel semántico; la sistematización, identificación e indexación de los contenidos argumentati$\operatorname{vos}^{67}$. Esencialmente se trata de proyectar algoritmos finalizados a la identificación de soluciones sobre la base de precedentes judiciales y otros elementos, según un esquema matemático, con la introducción de pruebas y elementos factuales como input, y la decisión final como output ${ }^{68}$.

No está de más precisar que elegir racionalmente significa tener en cuenta todos los datos pasados con el fin de prever eventos futuros con cierto grado de "certeza", aunque sería mejor hablar de "probabilidad"69.

La posibilidad de predecir el futuro es un deseo que desde siempre ha sido connatural al hombre. De hecho, la ciencia misma tiene como fin buscar leyes y fórmulas capaces de explicar los acontecimientos del mundo para así poder preverlos.

Sin embargo, el comportamiento humano y su connatural capacidad de elegir no son elementos determinables con base en las mismas leyes con las que se comprenden los fenómenos físicos o químicos, ya que el hombre es un producto muy especial de la evolución del planeta Tierra llamado homo sapiens, que se destaca por ese peculiar elemento que lo autodetermina de manera imprevisible: la libertad. Por tanto el comportamiento del hombre no es solamente el producto de sus estímulos bioquímicos o neurofisiológicos, sino también el resultado de las condiciones socioeconómicas o ambientales en las que vive ${ }^{70}$.

Ya a mitad del siglo xx, con la emergente cibernética, Lee Loevinger, jurista estadounidense, hablando de Jurimetría ${ }^{71}$ esperaba poder valerse de las ventajas de las técnicas electrónicas para resolver los problemas jurídicos, básicamente intentando reproducir, por medio de un calculador, los mecanismos intelectuales típicos de la

67 BICHI, R., Intelligenza artificiale e diritto, cit., 1773.

68 Véase TARUfFo, M., "Legalità e giustificazione della creazione giudiziaria del diritto", Riv. trim. dir. e proc. civ., 2001, 11 ss.

69 Mattera, R., Decisione negoziale e giudiziale, cit., 200.

70 Se conoce también lo influenciables que son las decisiones individuales y colectivas gracias a varias formas de nudging (THALER, R. y Sunstein, C. R., Nudge. La spinta gentile. La nuova strategia per migliorare le nostre decisioni su denaro, salute, felicità, Milano, Feltrinelli, 2018), es decir, "gentiles empujones" (cfr. BATTELl, E., "Il paternalismo giuridico libertario nella prospettiva dell'autonomia privata tra vincoli strutturali e limiti funzionali", Pol. diritto, 2018, 4, 579 ss.), a lo que se suma el preocupante fenómeno de las llamadas fake news, "sin mencionar las teorías relacionadas a la posverdad”: SimONCINI, A., L'algoritmo incostituzionale, cit., 79.

71 De este modo, QuARTA, E., "Giustizia e predizione: 1'algoritmo che legge il futuro", Giustizia insieme, 10 de marzo, 2019, recuerda que la jurimetría se concentra en: el almacenamiento, la localización electrónica de las informaciones jurídicas, la previsión de las decisiones jurídicas sobre la base del análisis de la conducta y la formalización del derecho y de la ciencia jurídica mediante la lógica simbólica. 
mente humana ${ }^{72}$, con el fin de alcanzar la previsión informatizada de los comportamientos en relación con la toma de decisiones por parte de los jueces ${ }^{73}$.

La exigencia de racionalizar la incertidumbre del futuro se traduce en la creación de una ciencia del razonamiento jurídico que va de la mano del antiguo sueño de predecir el futuro ${ }^{74}$. La "justicia predictiva" ha de entenderse como la posibilidad de prever el resultado de un juicio a través de algunos cálculos, en particular predecir la probable sentencia relativa a un caso específico, con el auxilio de algoritmos ${ }^{75}$.

Por un lado se trata, evidentemente, de un enfoque distinto del que suele denominarse "precedente judicial" 76 , por el otro, la llamada predictive coding, es decir, la justicia predictiva, está lejos de asimilarse al sistema de justicia al que hace referencia la famosa película Minority Report ${ }^{77}$.

La redefinición en términos racionales de la justicia "predictiva", así como la posibilidad de utilizar los métodos de la ciencia exacta en el ámbito jurídico para predecir eventos futuros, no puede dejar de relacionarse con el mito griego, específicamente con la diosa Diké, esto es "La Justicia" (que tenía que controlar las acciones de los hombres y sancionarlos por sus culpas ${ }^{78}$, pero más aún con el culto de Apolo en el templo de Delfos que, como es sabido, al menos hasta la época clásica, inducía a los antiguos griegos a dirigirse a tal isla para consultar a Apolo, que contestaba por medio de las palabras de la sacerdotisa Pitia. Fue solo a partir de la época romana que la función jurídica se reservó a una severa y especial clase y sus respectivos expertos, los "juristas" (término que, como es notorio, procede del latín), cuyas tareas se separaron de la religión, la moral y la misma política, permitiendo así una identificación autónoma que consintió que tal actividad se connotara como técnica en las manos de los sabios ${ }^{79}$.

72 En 1956, en un famoso congreso celebrado en Darmouth, se reunieron informáticos, psicólogos y filósofos con el fin de desarrollar un método interdisciplinario para estudiar la posibilidad de reproducir las actividades intelectuales propias del hombre a través de los calculadores. Cfr. TADDEI ELMI, G., Corso di informatica giuridica, Napoli, Simone, 2007, 87 s.

73 QuARTA, E., "L'algoritmo giuridico che realizza l'antico sogno dell'uomo di visualizzare scenari avveniristici: la giustizia nell'era 4.0", Personaedanno.it, 21 de febrero, 2019.

74 Belloso Martin, N., Algoritmos predictivos al servicio de la justicia, cit., 1-31.

75 Viola, L., "Giustizia predittiva”, www.treccani.it, Roma, 2018.

76 Passanante, L., Il precedente impossibile. Contributo allo studio del diritto giurisprudenziale nel processo civile, Torino, Giappichelli, 2018.

77 Basta recordar que en Estados Unidos, donde la predictive justice encontró una primera experimentación en la justicia penal, la predictividad intervino como un apoyo a la actividad de los jueces (Wisconsin Supreme Court, 13 luglio 2016, State vs. Loomis, case n. 2015AP157-CR, en 130 Harv. L. Rev. P. 1530). Cfr. De Miguel Beriaín, I., "Does the use of risk assessments in sentences respect the right to due process? A critical analysis of the Wisconsin v. Loomis ruling", Law Probability and Risk, 17, 1, 2018, 45-53; Freeman, K., "Algorithmic injustice: how the Wisconsin Supreme Court failed to protect due process rights in State v. Loomis", North Caroline Journal of Law and Techno$\log y$ (NCJL \& Tech.), 18, 2016, 75 ss.

78 Schiavone, A., “Ius”. L'invenzione del diritto in Occidente, Torino, Einaudi, 2005, 5 ss.

79 GiufFè̀, V., Il diritto dei privati nell'esperienza romana. I principali gangli, Napoli, Jovene, 2002. 
Sin duda alguna, cuando se habla de juez robot pareciera que se quisiera volver al modelo ideal de un "oráculo".

El uso del pasado para el futuro es un auxilio fundamental para la jurisdicción y también puede ayudar a los jueces en la inclusión de su decisión en el ámbito de los precedentes.

Ahora que fueron definidos los límites de la función jurisdiccional hipotéticamente asignada a la IA dotada de capacidad para emitir output en la forma de decisiones robóticas ${ }^{80}$, es necesario centrarnos en un aspecto que resulta cada vez más interesante en este campo: en lo que atañe a las exigencias de seguridad, como se especificó arriba, ciertamente hay que dudar de la existencia de máquinas infalibles.

Para incrementar el grado de seguridad de las decisiones es común que los expertos de la materia aseveren que es importante la posibilidad de instruir al juez robot introduciendo en su memoria todos los posibles "repertorios de jurisprudencia". De esta manera parecería posible contar con un dato objetivo e indiscutible, superando el ya citado subjetivismo que caracteriza tanto al juez humano como a la máquina proyectada para desempeñar las mismas funciones ${ }^{81}$.

Se ha discutido ampliamente acerca del orden y el peso de los criterios de interpretación como input necesarios para garantizar una mayor seguridad jurídica ${ }^{82}$.

Si bien es cierto que la restricción de la actividad interpretativa a modelos matemáticos podría llevar a consecuencias perjudiciales y, entre ellas, ante todo la prevalencia de un canon de interpretación estrictamente literal ${ }^{83}$, también hay que poner de relieve que, con una producción normativa tan policéntrica y reticular, a estas alturas el intérprete está obligado a reconstruir, mediar e inclusive identificar el correcto grado jerárquico y el grado de fuerza vinculante de la fuente, o la relación de prevalencia entre distintas fuentes en conflicto ${ }^{84}$.

Antes de continuar es preciso resaltar algunos límites estructurales de dicha hipótesis. El primer límite estaría representado por la delicada decisión sobre "cuándo" dejar de recoger datos. También sería difícil establecer cuándo la adquisición se haya completado, considerando que no se cuenta con una total digitalización de la inmensa producción jurídica, ni siquiera de las últimas décadas.

Existen otros límites ${ }^{85}:$ 1) Si los repertorios comprendieran también la jurisprudencia anterior ${ }^{86}$, el robot dispondría de un bagaje tan completo cuanto "superado",

80 Belloso Martin, N., Algoritmos predictivos al servicio de la justicia, cit., 8: "Los posibles usos de la inteligencia artificial en los sistemas de justicia".

81 Véase Castelli, C. y Piana, D., "Giustizia predittiva”, cit.

82 De Felice, M., "Calcolabilità e probabilità. Per discutere di 'incontrollabile soggettivismo della decisione"”, en AA.vv., Calcolabilità giuridica, cit., 43 ss.

83 Mattera, R., Decisione negoziale e giudiziale, cit., 207.

84 Manes, V., Il giudice nel labirinto. Profili di intersezioni tra diritto penale e fonti sovranazionali, Roma, Dikegiuridica, 2012, 20 ss.

85 LuCiani, M., La decisione giudiziaria robotica, cit., 887.

86 TARuffo, M., "Precedente e giurisprudenza", Riv. trim. dir. e proc. civ., 2007, 710 s. 
determinando posibles conflictos de instrucciones (p. ej., la jurisprudencia pre-republicana o la del período fascista). 2) Hipotéticamente, si se introdujera también la voluminosa jurisprudencia contemporánea los costes irían más allá del más mínimo respeto del principio de economía. 3) Si no se encuentran decisiones nomofilácticas de la Corte de Casación, o si existen pronunciamientos que entran en conflicto (incluso entre decisiones de las Secciones Unidas), sería necesario establecer un criterio para resolver el contraste, ya que no se podría recurrir al simple criterio cronológico que se aplica en la sucesión de las fuentes. 4) Vincular el juez robot a la jurisprudencia consolidada impediría la evolución misma de las orientaciones jurisprudenciales; además el resultado sería no tener la posibilidad de considerar el cambio de las exigencias sociales y de la sensibilidad de la comunidad humana en cuestión, sometiendo al juez ya no solo a la ley (art. 101 Constitución italiana), sino a la jurisprudencia. 5) Todo ello sería incompatible con la lógica de los ordenamientos de derecho romano, en los cuales no puede valer el "vínculo del precedente" 87. 6) Se menospreciaría notablemente el papel del abogado y del mismo arte del derecho, transmitido desde hace milenios en los actos y en la retórica forense ${ }^{88}$. 7) La motivación misma de la sentencia perdería su significado en la sentencia robótica, ya que el único motivo para que se efectuara sería garantizar su controlabilidad social o encontrar un posible error, ya sea informático o de ingeniería.

La respuesta no podría ser solo educar al juez robot para que adquiera las posiciones doctrinales y las praxis administrativas, ya que tendríamos otros problemas. De hecho, se trataría de establecer, al menos, qué peso atribuir a las tres distintas fuentes (jurisprudencia, doctrina, praxis) y entre las distintas praxis y doctrinas, incluyendo entre ellas las llamadas especulativas o más originales, que no representan una fuente de seguridad sino de dudas para considerar y solucionar.

Incluso en la literatura extranjera más apercibida, cuando se habla de riesgos concretos de la decisión robótica se tienen en cuenta, al menos, tres categorías de problemas: a) el carácter secreto o la inteligibilidad de la lógica que reside en el proceso de decisión, especialmente en el caso de los algoritmos de aprendizaje automá-

87 Holmes, O. W., "The path of the law”, Harvard Law Review 457, 1897 ss. La cuestión de la justicia predictiva se plantea de otra manera en los sistemas de common law, caracterizados por un indiscutible vínculo de proximidad entre la identificación del precedente y la adopción de la decisión del caso concreto: cfr. HARris, A., "Judicial decision making and computers", Vill. L. Rev., 12, 272, 1967, 312; cfr. asimismo LAWLOR, R. C., "What computers can do: analysis and prediction of judicial decisions", American Bar Association Journal, 49, 4, 1963, 337-344.

88 Véase Susskind, R., Tomorrow's Lawyers. An Introduction to Your Future, 2. a ed., Oxford, Oxford University Press, 2017, espec. 43 ss. y 184 ss. 
tico (problema de la black box) ${ }^{89}$; b) la aptitud discriminatoria del algoritmo ${ }^{90}$, y c) la humillación de la persona humana, objeto de decisiones totalmente automatizadas (problema de la dignidad) ${ }^{91}$.

Por lo tanto, si las decisiones robóticas no se controlan podrían consolidar las posiciones de privilegio, las desigualdades y las asimetrías que existen en una comunidad $^{92}$

Después de lo que se ha dicho sobre los riesgos y las críticas cabría preguntarnos, con el objeto de provocar una reacción, si sería posible que un robot, a través de su lógica binaria rígida, reconstruya una noción de "duda", "equidad" o "discrecionalidad" (como cuando se tiene que determinar una sanción entre un mínimo y un máximo), o si se quedaría en el mero nivel numérico-estadístico que, en todo caso, sería arbitrario ${ }^{93}$; sin considerar, además, las cláusulas generales ${ }^{94}$ y el uso de categorías e institutos que atañen a la conciencia social (p. ej., los conceptos de "moral pública" o de "diligencia del buen padre de familia") 95 .

Sin duda, hoy en día los robots (en cuanto máquinas inteligentes) pueden realizar con éxito una serie de actividades que requieren habilidades cognitivas que antes se consideraban demasiado difíciles de automatizar (p. ej., la IA aplicada a la conducción de vehículos) ${ }^{96}$. A la luz de lo que se ha dicho, la consideración más realista es que la justicia robótica no debería reemplazar la actividad del jurista, sino ponerse a su servicio en cuanto herramienta de trabajo.

Por lo tanto, el objetivo no debe ser limitar el razonamiento jurídico a una ecuación que, como se ha demostrado, dependería demasiado de las variables introducidas, por no mencionar más.

89 De "black box" hablan Pasquale, F., The Black Box Society: The Secret Algorithms that Control Money and Information, Cambridge-London, Harvard University Press, 2015; Kroll, J. A., "The fallacy of inscrutability", Phil. Trans. R. Soc., 376, 2133, 2018; Sнан, H., "Algorithmic accountability", Phil. Trans. R. Soc., 376, 2128, 2018; Pedreschi, D. et al., "Open the black box. Data-driven explanation of black box decision systems", ArXiv, 1, 2018, 1 ss.

90 Martínez García, I., "Inteligencia y derechos humanos en la sociedad digital", Cuadernos Electrónicos de Filosofía del Derecho, 2019, 40, 168-189; CHANDER, A., "The racist algorithm?", Michigan L. Rev., 115, 6, 2017, 1023-1045.

91 Noto La Diega, G., “Against the de-humanisation of decision-making”, jipitec, 9, 3, 2018.

92 Véase Baldwin, R., The Globotics Upheaval. Globalization, Robotics, and the Future of Work, London, Weidenfeld \& Nicolson, 2019; véase también Rodotà, S., Tecnologie e diritti, Bologna, Il Mulino, 1995.

93 De FinetTi, B., Teoria delle probabilità. Sintesi introduttiva con appendice critica, vol. I, Torino, Einaudi, 1970, 226.

94 Cfr., por todos, Mengoni, L., "Spunti per una teoria delle clausole generali", Riv. critica dir. privato, 1986, 5 ss.; Fabiani, E., Clausole generali e sindacato della Cassazione, Torino, utet, 2003, 712 ss.

95 Como recuerda Romano, B., Dalla metropoli verso internet, cit., 118: "los números son seguros, unívocos. Las palabras exigen una interpretación".

96 NAZZARo, A. C., "Macchine intelligenti ('smart cars'): assicurazione e tutela della 'privacy' - 'Smart cars insurance and protection of privacy", en Diritto del mercato assicurativo e finanziario, 2018, $1,77-93$. 
Desde esta perspectiva, el robot puede ser valioso, por ejemplo, para dirigir soluciones y para apoyar la fase de investigación en casos más sencillos y en aquellos basados en cálculos y evaluaciones de pronóstico. Pensemos en las llamadas cuestiones bagatelares o pequeñas causas (small claims), pero también en la determinación del daño en asuntos de responsabilidad civil (sobre todo en materia de circulación de vehículos) y el requerimiento de pago.

De hecho, se argumenta que la automatización del proceso de toma de decisiones, siempre y cuando esté bien elaborado, permite lograr ventajas significativas en términos de uniformidad, fiabilidad y capacidad de control de la decisión. Por lo tanto, la automatización parecería abstractamente alineada con los principios de eficiencia y economía de la acción judicial, respecto de procedimientos en serie basados en la presunción sobre la significativa probabilidad de un hecho determinado ${ }^{97}$.

Asimismo, la decisión del robot poco (o nada) procedería en el ámbito del derecho de familia y en asuntos relacionados con los derechos de las personas, tanto en materia de garantías procesales como de protección de los datos personales ${ }^{98}$.

En este sentido, no se puede pasar por alto el hecho de que un sistema basado en el respeto del precedente jurisprudencial favorece la consecución de la previsibilidad de las decisiones, corolario del principio de seguridad jurídica, en un plano de continuidad y coherencia" 99 .

Los conceptos de "seguridad jurídica" y "previsibilidad" (jurisprudencial) no significan un cálculo aritmético. De hecho, tanto las decisiones puntuales como el "derecho" en general se pueden calcular en cuanto constituyen una mera aplicación de la ley, o bien, tras verificarse el hecho descrito por la ley también debe verificarse el efecto jurídico, según lo establecido por la respectiva norma ${ }^{100}$. Este enfoque, en realidad, revela una necesidad que, más que de las reales necesidades humanas, surge de la economía moderna ${ }^{101}$.

Por lo tanto, cabe admitir que la pérdida de seguridad jurídica, tantas veces señalada en la doctrina, se debe principalmente a la falta de una jerarquía segura de las fuentes y a la presencia de una pluralidad de sujetos que hoy desempeñan un papel normativo diferente, aunque se trate de niveles que se distinguen en nacional, supranacional, comunitario, jurisprudencial, convencional, etc. (desde autoridades reguladoras nacionales hasta organizaciones internacionales, desde organismos locales hasta el parlamento nacional y europeo $)^{102}$. A lo anterior se suma un rol cada

97 Véase Resta, G., Governare l'innovazione tecnologica, cit., 213.

98 Véase PizzetTi, F., "La protezione dei dati personali e la sfida dell'intelligenza artificiale", en ID. (ed.), Intelligenza artificiale, protezione dei dati personali e regolazione, Torino, Giappichelli, 2018.

99 DAlfino, D., "Creatività e creazionismo, prevedibilità e predittività", Foro it., 2018, pt. v, c. 385 ss.

100 IRTI, N., Un diritto incalcolabile, cit.

101 IRTI, N., "Per un dialogo sulla calcolabilità giuridica, cit.; ID., "Capitalismo e calcolabilità giuridica (letture e riflessioni)", Riv. Società, 2015,801 ss.

102 Pino, G., "La gerarchia delle fonti del diritto. Costruzione, decostruzione, ricostruzione", Ars interpretandi, XVI, 2011, 53 ss.; MANEs, V., Il giudice nel labirinto, cit., el cual destaca cómo ahora el juez, 
vez más importante de entidades privadas nacionales y "globalizadas", tanto individuales como colectivas, algunas de las cuales se pueden ubicar en el plano del llamado soft law ${ }^{103}$.

Imaginar una justicia ejercitada por un juez robot, en modo tal de proceder a la simple y "mecánica” aplicación de la ley general y abstracta a un caso concreto ${ }^{104}$, significaría dibujar una falsa representación de la realidad ${ }^{105}$. Por ende, tal simplificación excesiva ${ }^{106}$ suscita perplejidad, considerando los actuales desafíos que plantea el ejercicio de la función judicial en la sociedad contemporánea.

Ahora bien, conviene reflexionar sobre las implicaciones de la efectividad de la protección judicial $^{107}$.

La actividad interpretativa, en cuanto ejercicio de la posibilidad de valorar, está naturalmente caracterizada por una cierta discrecionalidad ${ }^{108}$. Lo mismo ocurre cuando nos limitamos a identificar el significado -entre las varias opciones argumentables y plausibles- que se puede atribuir al texto, sin llegar a tomar parte en la formulación de la norma, teniendo en cuenta tanto el co-texto como el contexto ${ }^{109}$, e independientemente del enfoque subjetivo del intérprete ${ }^{110}$.

Adquiere relevancia, en este sentido, la crítica sobre la ampliación del ámbito de intervención de los jueces, con el riesgo de que estos últimos actúen como legum inventores ${ }^{111}$.

frente a una normativa "policéntrica y reticular", se ve forzado a una continua labor de reconstrucción y mediación en un "laberinto", determinando así una acentuación de su discrecionalidad y creatividad en decidir también "qué es fuente y determinar caso por caso el grado preceptivo" (p. 19 s.).

103 Véase CAFAGGI, F., "Regolazione transnazionale e trasformazioni dello Stato: un'introduzione”, en Regolazione transnazionale e trasformazioni dello Stato, Bologna, Il Mulino, 2016. 11-55. Sobre el tema cfr. Ferrarese, M. R., "Decentramento e privatizzazione: due svolte 'critiche' per il diritto attuale”, en Luminoso, A. (ed.), Diritto e crisi, Milano, Giuffrè, 2016, 9-21; ID., Diritto sconfinato, Roma-Bari, Laterza, 2006.

104 Reflexión expresada hace ya un siglo, entre otros, por PACCHIONI, G., "I poteri creativi della giurisprudenza", Riv. dir. comm., 1912, 41 ss.: "El juez no es mero instrumento de aplicación mecánica del contenido de la ley: él es intérprete. E interpretar significa explicar, adaptar, completar".

105 IRTi, N., "La crisi della fattispecie", Riv. dir. proc., 2014, 43 ss.

106 Véase De Felice, M., "Su probabilità, 'precedente' e calcolabilità giuridica”, en CARLEO, A. (ed.), Il vincolo giudiziale del passato. I precedenti, 2018, 37 ss.; RuLLI, E., "Giustizia predittiva, intelligenza artificiale e modelli probabilistici. Chi ha paura degli algoritmi?”, Analisi giuridica dell'economia, 2018, 533 ss.

107 Para una exhaustiva reconstrucción de las configuraciones dogmáticas sobre la interpretación de la ley con el fin de garantizar el derecho de una protección judicial efectiva, cfr. VetTori, G., s.v. "Effettività delle tutele (dir. civile)", Enciclopedia del diritto, Milano, Giuffrè, 2017, 381-407.

108 Cass., Sez. Un., 11 julio de 2011, n. 15144, en Giusto processo civ., 2011, 1117 ss., con nota de F. AulETtA: "la norma, una vez en vigor, no permanece cristalizada en sí misma, sino que está sujeta, ex se, a tendencias evolutivas".

109 Gentili, A., Senso e consenso, iI, cit., 492 ss.

110 Dalfino, D., Creatività e creazionismo, cit., c. 388.

111 Véanse Castronovo, C., Eclissi del diritto civile, Milano, Giuffrè, 2015: "las decisiones ya no pueden ser justificadas por medio de comprobados elementos pre-existentes de carácter normativo o fáctico sino, en definitiva, a través de las consecuencias"; Nuzzo, M., "Il problema della prevedibi- 
Así las cosas, el modo correcto de concebir la predictibilidad es la capacidad de previsibilidad de las decisiones en el marco de la seguridad jurídica ${ }^{112}$, no tanto como la "adquisición preventiva" de un resultado ${ }^{113}$, sino más bien como la "expectativa razonable". Por consiguiente, la posible sentencia emitida en cada caso específico no representa una especie de justicia anticipada, sino que en últimas constituiría un juicio de pronóstico basado en el razonable resultado de un proceso ${ }^{114}$.

Entonces, a la tecnología le corresponde ofrecer apoyo y ayuda para que los seres humanos tomen decisiones, sin llegar a reemplazarlos en un ámbito tan delicado como el ejercicio de la función judicial ${ }^{115}$. A fin de cuentas, el desafío se centra en solucionar algunos de los problemas planteados a través de las matemáticas, sin que esta ciencia se ponga en el lugar de quien juzga, sino que lo apoye ${ }^{116}$.

\section{Experiencias y modelos probabilísticos en el marco de la justicia robótica}

Una experiencia muy exitosa en tiempos recientes es la plataforma holandesa llamada Rechtwijzer uit elkaar (guía legal sobre la separación), que brinda asistencia a las partes en el juicio de separación. A finales de 2016, más de mil parejas habían utilizado dicha plataforma ${ }^{117}$.

lità delle decisioni: calcolo giuridico secondo i precedenti”, en AA.vv., Calcolabilità giuridica, cit., 142; Di Porto, A., "Calcolo giuridico secondo la legge nell'età della giurisdizione”, ibíd., 119 ss .

112 Véase, Costantino, G., "Giustizia predittiva e prevedibilità della giurisprudenza: note introduttive", ponencia en la Conferencia internacional de estudios "Giustizia predittiva e prevedibilità delle decisioni", cit.

113 Confróntese con Viola, L., L'interpretazione della legge con modelli matematici, cit., $167 \mathrm{~s}$.

114 Véase por su actualidad Poisson, S. D., Recherches sur la probabilité des jugements en matière criminelle et en matière civile, Paris, 1837; DE CONDORCET, M., Essai sur application de l'analyse à la probabilité des décisions rendues à la pluralité des voix, Paris, 1785.

115 Mattera, R., Decisione negoziale e giudiziale, cit., 206.

116 La tesis sostenida por ViolA, L., "L'interpretazione della legge ex art. 12", cit., se resume de la manera siguiente: - el artículo 12 de las Preleyes del código civil italiano establece una regla, de carácter matemático, para resolver los conflictos interpretativos, desplegando no solo los posibles tipos de interpretación sino también la posible prevalencia de uno sobre el otro (p. ej., legitimando la interpretación analógica solo de manera subsidiaria respecto de la interpretación literal); - las proposiciones de la ley son algoritmos ya que están construidas según el modelo si... entonces: si se verifica el hecho $\mathrm{x}$, entonces se seguirá la sanción y; - este proceso es un modelo matemático dado que el pronunciamiento en un caso concreto está determinado por la composición entre hecho y derecho (pronunciamiento $=$ hecho $\circ$ derecho), en la medida en que la "síntesis" entre el hecho específico (con las debidas pruebas) y las normas indicadas es lo que determina el contenido de la decisión; las cláusulas presentes en la legislación tienen que ser interpretadas según los criterios del artículo 12 de las Preleyes, que es aplicable de manera general, alineándose a los principios generales de la legislación, incluidos los constitucionales, con la consecuencia de que la interpretación final nunca podrá ser extra legem, sino intra ius; - el derecho, concebido como mundo de las normas, tiene que ser calculable y objetivo, con el fin de permitir el control (de acuerdo con la tradición romanística).

117 Véase la página web: https://rechtwijzer.nl/uit-elkaar. 
También hay modelos experimentales cuyo objetivo es desafiar las jurisdicciones consolidadas, como el algoritmo desarrollado para predecir las sentencias (más bien, para llegar a la misma decisión, basándose en la misma información) del Tribunal Europeo de Derechos Humanos, con un grado de precisión cercano al $80 \%{ }^{118}$.

De hecho, están aumentando los estudios destinados a llegar a conclusiones similares a las de los tribunales formados por jueces humanos. Ejemplo de ello es la experiencia de los académicos estadounidenses que declaran haber predicho el $67 \%$ de las decisiones de los tribunales de bancarrota, a pesar de contar con muy poca información ${ }^{119}$; otros modelos experimentales han anticipado el $82 \%$ de las decisiones en materia de inmigración y asilo ${ }^{120}$.

Todos estos ejemplos muestran que es cada vez más sutil la frontera entre los algoritmos de apoyo al trabajo de los jueces y los que sugieren una decisión. Por tal razón, no obstante podamos convenir en que la idea de abandonarse a la justicia actuarial procedente de las nuevas tecnologías es preocupante, parece irracional negarse a considerar un fenómeno que amerita ser mayormente conocido y regulado ${ }^{121}$.

Vale la pena mencionar que en la vecina España, una multinacional que opera en el ámbito editorial jurídico promovió en tiempos recientes una herramienta de análisis jurisprudencial estadístico y predictivo ${ }^{122}$ : "Jurimetría te ofrece respuestas a preguntas complejas, por ejemplo: [...] ¿Cuántas posibilidades de éxito tengo? ¿Cuál es la mejor manera de argumentar este caso concreto [...]?", asimismo, "Jurimetría le proporcionará indicadores, tendencias y pronósticos de los resultados de un caso jurídico concreto. Gracias al análisis y a la clasificación de millones de documentos, al extraer datos clave, como la parte vencedora en la parte resolutiva, la entidad de la condena, el tribunal, entre muchos otros, [...] obtendrás respuestas claras sobre la posibilidad de éxito de tu caso y la probable duración del juicio" ${ }^{23}$; hay que tener en cuenta el empleo de palabras como: "probable", "probabilidad", "posibilidad", "tendencias" y "pronósticos".

118 Aletras, N. et al., Predicting Judicial Decisions of the European Court of Human Rights: a Natural Language Processing Perspective, en Peer Journal of Computer Science, 2:e93, 24 de octubre, 2016.

119 Ash, E. et al., Judge Writing Style Predicts Decision Tendencies in U.S. Bankruptcy Courts, Technical Report, 2018.

120 Chen, D. L. y J. EAgel, "Can machine learning help predict the outcome of asylum adjudications?", en Proceedings of the Association for Computing Machinery Conference on Artificial Intelligence and the Law, 2016.

121 Rulli, E., Giustizia predittiva, cit., 537.

122 Sobre este punto se hace referencia prácticamente textual al texto de De La Oliva SANTos, A., "Giustizia predittiva", cit., 888-889, el cual señala cómo, en España, a partir de la reforma de 1974, el código civil (1889) en su título preliminar contiene un conjunto de disposiciones calificadas como casi-constitucionales por algunos juristas autorizados, en particular el artículo 3, cuyo tenor recuerda el artículo 12 de las Preleyes del código civil italiano.

123 Ibíd., 884. 
Esta experiencia es muy interesante en cuanto nos permite detectar que el empleo del adjetivo "predictivo" en la literatura es inapropiado, por las razones explicadas anteriormente, considerando que los modelos algorítmicos mencionados no predicen el futuro, pero sí ofrecen una serie de posibles resultados ${ }^{124}$.

Está claro que la evolución tecnológica que acabamos de describir está provocando una asimilación de facto entre los sistemas de common law y de civil law, acercando a este al primero ${ }^{125}$.

Merece la pena mencionar, a estas alturas, la decisión del Tribunal Constitucional francés (Conseil constitutionnel) n ${ }^{\circ} 2018-765$ del 12 de junio de $2018^{[126]}$, que reconoció que la Administración Pública puede utilizar algoritmos, incluso en la adopción de decisiones que afectan a una persona solamente. Se especificó que cada vez que se confíe a un algoritmo la toma de una decisión es necesario establecer de antemano las reglas y los criterios que serán aplicados. Si esto se respeta, adoptando un mecanismo de control humano ex ante, no se podrá alegar que se trate de una renuncia de la administración al ejercicio de su poder regulador.

Con dicha premisa, el Tribunal ha identificado tres límites para el uso legítimo de algoritmos en las decisiones: (i) es necesario señalar expresamente si la decisión fue tomada en virtud de un algoritmo y, bajo solicitud de la parte interesada, la administración debe poder explicar las principales características del funcionamiento del respectivo algoritmo; (ii) el uso exclusivo de un algoritmo está prohibido si la decisión se refiere a datos personales sensibles, como el origen étnico; las creencias religiosas, políticas o filosóficas; la afiliación a sindicatos, así como otros datos relativos a la salud, datos genéticos o biométricos, o aquellos relacionados con la inclinación sexual de las personas; (iii) la posibilidad real de que el encargado del tratamiento de los datos pueda controlar el proceso de elaboración del algoritmo, para poder explicar a los destinatarios de las medidas, de manera detallada, clara e inteligible, que la decisión se tomó de conformidad con la ley ${ }^{127}$.

De lo dicho se deduce que, si estos principios se aplicaran también en el campo jurisdiccional, el resultado sería excluir la mayoría, o incluso todos los procesos

124 Cabe señalar la institución de la plataforma online "predictice.com" en Francia, con el fin específico de permitir el cálculo de las probabilidades de éxito de un proceso, a partir de un cálculo estadístico realizado con datos de carácter general y metadatos relativos al caso específico, recurriendo al uso de algoritmos predictivos. Véase Martinay, A. y Mazens, M., Regards sur les "promesses" de la justice predictive, [en línea], disponible en: http://blogs.univ-poitiers.fr/c-marcon; DONDERO, B., La justice prédictive, [en línea], disponible en: https://brunodondero.com/2017/02/10/la-justice-predictive, 2017; cfr. asimismo GARAPON, A., "Les enjeux de la justice prédictive", Le semaine juridique, éd. générale, Hebdomadaire, n. 1-2, 2017.

125 Véase Tiscornia, D., Il diritto nei modelli dell'intelligenza artificiale, Bologna, CLuEB, 1996, según la cual en la formalización del conocimiento jurídico la distinción es entre sistemas de normas (civil law) y sistemas basados en casos (common law). Mientras que los primeros parecen más adecuados al conocimiento legislativo, los otros respondes más a la idea de IA.

126 Disponible en: www.conseil-constitutionnel.fr/decision/2018/2018765DC.htm

127 Véase SASSI, S., "Gli algoritmi nelle decisioni pubbliche tra trasparenza e responsabilità", Analisi Giuridica dell'Economia, 2019, 1, 109 ss., esp. 116 ss. 
penales. En asuntos administrativos, los juicios relacionados con los derechos de la personalidad y la ciudadanía sin duda quedarían fuera del conocimiento del algoritmo. En el contexto de la jurisdicción civil no podría someterse a la utilización del algoritmo una parte importante de los procedimientos de derecho familiar, laboral y sindical, de seguros y de indemnización por daños biológicos, aunque precisamente en estos casos tendría una utilidad indudable ${ }^{128}$.

Es interesante observar que el Tribunal Constitucional francés dictó unas condiciones tan restrictivas solo para los casos cuya decisión sea tomada exclusivamente por un algoritmo. Cabe resaltar este aspecto muy claramente, ya que si el algoritmo representa un instrumento de ayuda para la decisión del juez, los límites mencionados resultarían superados o, al menos, superables.

Más allá de cualquier legítima sospecha, las nuevas tecnologías de robots y algoritmos pueden proporcionar herramientas valiosas para optimizar el trabajo de abogados y jueces en la selección de la información necesaria para establecer estrategias de decisión ${ }^{129}$.

Siendo puntuales, ya existe un programa llamado Case Francia Alfa, desarrollado por estudiantes de Cambridge, que ha desafiado a 100 juristas procedentes de los mejores despachos de abogados a tomar decisiones en materia de indemnización de daños, en casos de seguros o de protección del crédito. De 775 casos, el programa acertó el resultado en un $88,6 \%$, mientras que los juristas solamente lo hicieron en el $66,3 \%$.

En tiempos más recientes se han experimentado el programa de algoritmos CoIn - Contract Intelligence, para interpretar acuerdos comerciales, y el sistema KIRA, para evaluar las cláusulas abusivas en los contratos. A partir de estas experiencias queda claro cómo los potenciales de aplicación involucran, teniendo en cuenta los límites expuestos, sectores muy diferentes (seguros, derechos humanos, contratos), pero igualmente caracterizados por una fase de previsión en la que resulta fundamental el estudio y el análisis de los antecedentes.

No es una casualidad que precisamente en Francia hayan surgido start-up que ofrecen las primeras soluciones para el cálculo probabilístico de las decisiones en el ámbito civil ${ }^{130}$. Son compañías que producen software y, en general, ofrecen servicios a otras empresas o despachos de abogados, aunque nada impide que el destinatario sea la administración de justicia, para proporcionar "ayuda" al trabajo del juez.

128 AnConA, M., "Verso una giustizia predittiva mite", ponencia en el "Convegno internazionale di studi Giustizia predittiva e prevedibilità delle decisioni”, cit., ahora en: https://tecnojustitaly.wordpress. com/; ID., "Industria 4.0 e sistema giustizia", Caos. Rivista quadrimestrale per l'innovazione sociale, 2017, vol. 2 .

129 Véase LAmON, B., "La profession d'avocat et la justice prédictive: un bel outil pour le développement du droit", Récueil Dalloz, 14, 2017.

130 Rulli, E., "Giustizia predittiva”, cit., 542. 
Por otro lado, es necesario recordar que la misma Francia ha modificado recientemente (con la Ley n. ${ }^{\circ} 2018-493$ del 20 de junio de 2018) ${ }^{131}$ el artículo 10 de la Ley Informatique et libertés de 1978, relativa a la informática, a los archivos y a las libertades, estableciendo los siguientes límites y $\operatorname{principios}^{132}$ : a) Ninguna decisión judicial que implique una evaluación del comportamiento de una persona puede basarse en el procesamiento automatizado de datos personales destinados a evaluar ciertos aspectos de la personalidad del individuo. b) Toda decisión que produzca efectos legales con respecto a una persona y que esté basada en el procesamiento automatizado de datos personales debe necesariamente observar las condiciones establecidas por el artículo 22 del RGPD. c) Decisiones administrativas individuales basadas en el procesamiento automatizado de datos son admisibles, siempre que el procesamiento no se relacione con categorías particulares, como datos genéticos, de salud o sobre preferencias políticas, etc. d) Estas decisiones deben incluir la mención explícita del procesamiento automatizado; para estas decisiones, el responsable de los datos debe garantizar la plena comprensión del procesamiento algorítmico. e) Cualquier decisión por la cual la administración decida sobre un recurso administrativo no puede tomarse únicamente sobre la base de un procesamiento automatizado de datos personales ${ }^{133}$.

Sin rechazar la oportunidad de tales servicios tecnológicos a priori ${ }^{134}$, dada la delicadeza y la relevancia constitucional ${ }^{135}$ del impacto de la robótica en el sistema de "justicia" y considerando las funciones de garantía de la Administración Pública en general, la cuestión que razonablemente debe plantearse es si dichas actividades deberían quedar sujetas a un sistema de vigilancia pública "especializada" o, al menos, a estrictos requisitos de autorización.

El tema de la regulación normativa del fenómeno en cuestión no solo conlleva varios problemas, sino que además es blanco de las críticas de quienes temen limitar el rápido desarrollo tecnológico a través de normas demasiado estrictas, que podrían quedar obsoletas en poco tiempo.

Por lo tanto, parece fundamental desarrollar una infraestructura institucional eficaz, que incluya normas, remedios y procedimientos adecuados a los intereses en

131 Gaudemet, P. Y., "La justice à l'heure des algorithmes", Rev. Dr. Pub., 3, 2018, 651 ss.; Pauliat, H., "La décision administrative et les algorithmes: une loyauté à consacrer", Rev. Dr. Pub., 3, 2018, 641 ss.

132 Resta, G., "Governare l'innovazione tecnologica", cit., 228 s.

133 Véase DuclercQ, J.-B., "Le droit public à l'ère des algorithmes", Rev. Dr. Pub., 5, 2017, 1401 ss.; MeкKi, M., "If code is law, then code is justice? Droit et algorythmes", Gazette du Palais, 27.6.2017.

134 Véase SolAR CAYÓN, J. I., La inteligencia artificial jurídica. El impacto de la innovación tecnológica en la práctica del derecho y el mercado de servicios jurídicos, Cizur Menor (Navarra), Aranzadi, 2019.

135 Véase Casonato, C., "Intelligenza artificiale e diritto costituzionale: prima considerazioni", Dir. pubbl. comp. eur., 2019, 101 ss. y Simoncini, A. y Suweis, S., "Il cambio di paradigma nell'intelligenza artificiale e il suo impatto sul diritto costituzionale", en Riv. filosofia dir., 1, 2019, 87 ss. 
juego y que pueda garantizar un control adecuado sobre la adopción de decisiones robóticas.

El Parlamento Europeo, por su parte, aprobó una resolución que contiene recomendaciones específicas, insistiendo en la adopción de normas que regulen el fenómeno que se está analizando ${ }^{136}$. Se trata de la Resolución del 16 de febrero de 2017, titulada "Normas de Derecho civil sobre robótica"137, cuya lectura refleja la situación actual de la ley en materia de robótica y que, en todo caso, deberá atenerse a la preservación de algunos principios fundamentales como la protección de la seguridad y la salud humana, al igual que la integridad y la dignidad de las personas, el respeto de la vida privada, de la autodeterminación y el principio de no discriminación, además de la protección de datos personales ${ }^{138}$.

El asunto es que incluso cuando el algoritmo se pueda conocer y comprender, y aunque no represente el único fundamento de la decisión, puede ser discriminatorio en sí mismo ${ }^{139}$. Esto ocurre cuando el algoritmo predictivo se construye a partir de datos que son discriminatorios a priori, ya que un algoritmo reflejará siempre la calidad de los datos en los que se funda ${ }^{140}$.

Es por esto que la legislación de la Unión Europea, especialmente en asuntos financieros y bancarios, por un lado, ha fomentado el empleo de formas invasivas de creación de perfiles de clientes, con el fin de tomar decisiones basadas en la evaluación de la solvencia y del mérito crediticio ${ }^{141}$ (los llamados mecanismos de credit

136 Véase PALmerini, E. et al., "RoboLaw: Towards a European Framework for robotics regulation", en Robotics and Autonomous Systems, 2016, 78 ss.

137 SAssi, S., Gli algoritmi nelle decisioni pubbliche, cit., 111 ss.

138 MATtera, R., Decisione negoziale e giudiziale, cit., $198 \mathrm{s.}$

139 Tegmark, M., Vita 3.0. Essere umani nell'era dell'intelligenza artificiale, Milano, Raffaello Cortina, 2018, 145, señala que "un estudio de 2016 ha afirmado efectivamente que el software para la previsión de la reincidencia usado en Estados Unidos estaba predispuesto contra los afroamericanos y había contribuido a la emanación de sentencias desiguales".

140 En el considerando n. ${ }^{\circ} 71$ del Reglamento 679/2016 existe un principio fundamental según el cual, "considerando las circunstancias y el contexto específico en el que se tratan los datos personales, es oportuno que el titular del tratamiento utilice procedimientos matemáticos o estadísticos apropiados para la elaboración de perfiles, aplique medidas técnicas y organizativas adecuadas con el fin de garantizar, sobre todo, que los factores que implican imprecisiones de los datos se rectifiquen y que sea minimizado el riesgo de errores, para así garantizar la seguridad de los datos personales mediante un método que considere los eventuales riesgos que amenazan los intereses y los derechos del titular, impidiendo además efectos discriminatorios hacia las personas físicas por motivos relacionados con su raza o etnia, opiniones políticas, religión o convicciones personales, afiliación sindical, genética, su estado de salud u orientación sexual” (Reg. GDPR, considerando n. ${ }^{\circ}$ 71). El artículo 71 del Reglamento 679/2016 disciplina el denominado tratamiento automatizado, destinado a la elaboración de los datos personales, pero el tema abarca cualquier forma de algoritmo predictivo. Así lo asevera Simoncini, A., "L'algoritmo incostituzionale", cit., 84.

141 Véase el considerando n. ${ }^{\circ} 27$ y el artículo 8 de la Directiva 2008/48/CE, sobre los contratos de crédito al consumidor, el cual prescribe: "Member States shall ensure that, before the conclusion of the credit agreement, the creditor assesses the consumer's creditworthiness on the basis of sufficient information, where appropriate obtained from the consumer and, where necessary, on the basis of a consultation of the relevant database. Member States whose legislation requires creditors to assess 
scoring para calcular la fiabilidad financiera en el marco de los procedimientos de financiación para particulares y empresas) ${ }^{142}$; y por el otro lado, ha requerido que los datos utilizados se caractericen por un alto grado de "calidad", al ser puntuales, exactos y detallados ${ }^{143}$.

Incluso la sucesiva Comunicación de la Comisión Europea del 25 de abril de 2018 sobre "Inteligencia artificial para Europa" ${ }^{144}$ se centra en poner la IA al servicio del hombre ${ }^{145}$. De hecho, tras definir la IA como un conjunto de "sistemas que manifiestan un comportamiento inteligente, pues son capaces de analizar su entorno y pasar a la acción -con cierto grado de autonomía- con el fin de alcanzar objetivos específicos" ${ }^{146}$, aclara que la Unión Europea tiene que garantizar un marco ético y jurídico que tutele los derechos y las libertades fundamentales de los ciudadanos en el plano económico y social y que respete los principios de transparencia y responsabilidad $^{147}$.

Se podría mencionar, por último, la Carta Europea sobre el Uso Ético de la Inteligencia Artificial en los Sistemas Judiciales y su Entorno, aprobada en la sesión plenaria del 3 de diciembre de 2018 en Estrasburgo, por parte de la Comisión Europea para la Eficiencia de la Justicia (CEPEJ). La Carta proporciona directrices para que los operadores del derecho elaboren y apliquen tecnologías avanzadas en el ejercicio de la función judicial. Se mencionan especialmente la exigencia de tutela y garantía de los derechos fundamentales de la persona, la exclusión de soluciones y prácticas que

the creditworthiness of consumers on the basis of a consultation of the relevant database may retain this requirement"; cfr., además, el Consumer Financial Services Action Plan de la Comisión de 2017.

142 Keats Citron, D. y Pasquale, F., “The Scored Society: Due Process for Automated Predictions", en Washington Law Review, 89, 1, 2014, 1-33.

143 Resta, G., Governare l'innovazione tecnologica, cit., 208, especifica: "En este contexto se prevé que los datos deben ser exactos y actualizados si es necesario, no pueden estar almacenados durante un periodo excesivo de tiempo, y de todas formas deben ser accesibles, controlados, rectificados, integrados e incluso cancelados a petición del sujeto pertinente (arts. 13-21 GDPR). Esto implica, en pocas palabras, que la infraestructura regulatoria se caracteriza por una serie de filtros capaces de elevar la calidad del ecosistema informativo, seleccionando ex ante tipología, volumen y caracteres de las informaciones útiles para la elaboración de perfiles, para un análisis predictivo y para decisiones algorítmicas. Esto representa una circunstancia considerable porque, como se sabe, los algoritmos funcionan si son apropiados, exactos y actualizados, de lo contrario se corre el riesgo de producir decisiones poco fiables".

144 Comunicación de la Comisión al Parlamento Europeo, al Consejo, al Comité Económico y Social Europeo y al Comité de las Regiones, L'Intelligenza artificiale per l'Europa, Bruxelles, 25.4.2018, com (2018)237 final. Asimismo Scherer, M. U., "Regulating artificial intelligence systems: risks, challenge, competences, and strategies", Har. J.L. \& Tech., 29, 2016, 354 ss.

145 Véanse los recientes estudios llevados a cabo respectivamente por la Comisión y por el Parlamento Europeo: Craglia, M. (ed.), Artificial Intelligence. A European Perspective, Luxembourg, 2018; Sartor, G., Artificial Intelligence: Challenges for EU Citizens and Consumers, Brussels, PE $631.043,2019$.

146 SAuvÉ, J.-M., Le juge administratif et l'intelligence artificielle, cit., 1.

147 Temme, M., "Algorithms and transparency in view of the general data protection regulation", Eur. Data Prot. L. Rev., 3, 4, 2017, 473-485. 
induzcan a fenómenos discriminatorios y la adopción de criterios de transparencia y "equidad" 148 .

\section{Conclusiones}

El uso de algoritmos por parte de computadores particularmente avanzados no puede, por la naturaleza misma de la ciencia matemática subyacente, sustituirse a la sensibilidad, percepción y pensamiento humano, necesarios para la interpretación de la ley ${ }^{149}$.

Es menester concluir que los individuos no pueden y no deben ser sometidos a una decisión totalmente automatizada. No obstante, toda vez que el ordenamiento admitiera que así ocurriera, se tendrían que aplicar, como mínimo, algunas garantías sustanciales, como la reconsideración de la decisión robótica, a través de una participación humana directa; además, obviamente, de la constatación relativa a la conformidad del algoritmo a los requisitos de la ley ${ }^{150}$.

Ciertamente, no se puede negar que la confianza en la robot-justice, como se ha dicho al principio, se debe a la crisis por una grave incertidumbre que reina en detrimento de la sociedad y a la crisis de la doctrina de la interpretación e incluso de la jurisdicción misma.

Por consiguiente, es razonable pensar que la justicia robótica, en cuanto justicia predictiva, conlleva las mismas limitaciones de cualquier cálculo de probabilidades, con la diferencia de que una previsión no es lo mismo que una predicción ${ }^{151}$; por lo que, cuando exigimos que las decisiones judiciales sean previsibles, en realidad se manifiesta respecto del juez una pretensión de seguridad jurídica que, desde el punto de vista ontológico, no puede considerarse par a la infalibilidad matemática ${ }^{152}$.

Con respecto a la "justicia predictiva", hay que considerar además que cuanto más seguro y previsible sea el resultado de un posible juicio, más probable será también que se intente resolver el asunto sin acudir a los tribunales, en últimas recurriendo a procedimientos de justicia alternativos (ya sean Alternative Dispute Resolution - ADR u Online Dispute Resolution - ODR, en las formas contempladas por

148 Véase MArcoz, G., "Intelligenza artificiale e giustizia, perché è importante la Carta etica europea", Agenda digitale.eu, 15 de febrero, 2019. Véase también "Codice Etico UE sull'intelligenza artificiale: forte la tecnica, debole la politica", Focus.it, 2 de enero, 2019; Morelli, C., "Algoritmi e diritti umani: si rischia la collisione (immaginate a danno di chi?)", Altalex.com, 9 de julio de 2018. Véase asimismo RufFolo, U., "Per i fondamenti di un diritto della robotica self-learning; verso una responsabilità da algoritmo?", 13 ss. y AMIDEI, A., "Robotica intelligente e responsabilità: profili e prospettive evolutive del quadro normativo europeo", 63 ss., ambos en RufFolo, U., Intelligenza artificiale e responsabilità, Milano, Giuffrè, 2018.

149 De la Oliva Santos, A., "Giustizia predittiva", cit., 883 ss.

150 Resta, G., Governare l'innovazione tecnologica, cit., 235.

151 De Felice, M., "Calcolabilità e probabilità", cit., 54.

152 Douglas Price, J. E., La decisión judicial, cit., passim; De Finetti, B., Teoria delle probabilità, cit., I, 33 . 
los diferentes sistemas jurídicos); ya que, cuando el resultado del juicio se considera probable, las partes razonablemente estarán más dispuestas a llegar a un acuerdo, ahorrando así tiempo y conteniendo los gastos ${ }^{153}$, de manera que discutan directamente el quantum y no el si [an] (que se da por sentado). Esto vale también para el arbitraje porque, al usar modelos matemáticos se puede reducir el riesgo de parcialidad a la vez que se gana mayor velocidad y transparencia.

Lo mejor sería, entonces, no referirse a la justicia predictiva en términos de previsión del futuro basada en fórmulas matemáticas (nuevas sustitutas de las antiguas fórmulas mágicas), sino como la previsión razonable de una decisión jurisdiccional a través de la correcta identificación e interacción de las variables interpretativas de los hechos y las normas. Esto sin la pretensión del supuesto de "seguridad jurídica", que no puede ni debe tener, sino con la claridad de que cuenta con las ventajas y las limitaciones que, en todo caso, no la vuelven mejor, comparada con los tradicionales procedimientos judiciales confiados a hombres y no a robots ${ }^{154}$. Vale la pena subrayar este aspecto en el sistema jurídico italiano, que ya expresamente prevé y disciplina la llamada "negociación robótica" en el artículo 1, inciso 495 de la Ley n. ${ }^{\circ}$ 228/2012 (llamada Ley de Estabilidad de 2013) ${ }^{155}$.

Se señala también la reforma de 2016 que sustancialmente transformó el juicio civil de legitimidad, estableciendo que los precedentes que constituyen la jurisprudencia de la Corte de Casación tienen por ley una naturaleza vinculante, atendiendo lo dispuesto en el artículo 360 bis c.p.c. La Corte Suprema, en la Sección vi Civil, sentencia n. $.^{\circ} 7155 / 2017$, dio nueva relevancia a la inadmisibilidad prevista en el artículo 360 bis, con respecto a los casos del carácter manifiestamente fundado o infundado del recurso, al que hace referencia el artículo 375, numeral 5 c.p.c., por lo que se puede afirmar que el stare decisis ha sido acogido también en Italia ${ }^{156}$. Y esto, hay que decirlo, favorece el posible uso de la robótica aplicada a la justicia.

En el ámbito del derecho procesal civil por lo menos puede esperarse que la previsibilidad de un juicio produzca un efecto virtuoso respecto de la demanda de justicia, de manera que pueda reducir gradualmente los casos no fundados. Por otro

153 Viola, L., "L'interpretazione della legge ex art. 12”, cit.

154 Véase Olhede, S. C. y Wolfe, P. J., "The growing ubiquity of algorithms in society: implications, impacts, and innovations", Phil. Trans. R. Soc., 2018, 1-15.

155 La norma estipula: "Se considera actividad de negociación de alta frecuencia aquella actividad generada por un algoritmo informático que determina de manera automática las decisiones relacionadas con el envío, la modificación o la cancelación de las órdenes y de los parámetros correspondientes". A su vez, se señala el decreto legislativo del 24 de febrero de 1998, n. ${ }^{\circ} 58$, que contiene el Testo Unico Bancario (тUв), el cual dispone: 'La 'negociación algorítmica' se refiere a la negociación de instrumentos financieros cuyo algoritmo informatizado determina automáticamente los parámetros individuales de las órdenes, como por ejemplo el momento en el que inicia, el plazo correspondiente, el precio, la cantidad o las modalidades para administrar la orden luego del envío, con una mínima o inexistente intervención humana, excluyendo los sistemas utilizados únicamente para transmitir órdenes a una o más sedes de negocio, para abordar órdenes que no implican la determinación de parámetros de negociación, para confirmar órdenes o para cumplir el reglamento de las operaciones".

156 Mattera, R., Decisione negoziale e giudiziale, cit., 204. 
lado, los magistrados podrían beneficiarse al ser colocados en las mejores condiciones para decidir con mayor conciencia en aquellos casos en los que asumirán la responsabilidad de un cambio en la jurisprudencia ${ }^{157}$.

En el momento actual, como se explicó, los numerosos riesgos y obstáculos que se encuentran en el camino hacia un sistema basado en la "predictividad" de las decisiones robóticas parecen prevalecer sobre los beneficios ${ }^{158}$. Sin embargo, los horizontes que aparecen gracias a las nuevas tecnologías, consideradas como herramientas de apoyo a la actividad del juez y sin que se sustituyan a él en la toma de la decisión (como en el caso del "juez automático" o "juez robot”) ${ }^{159}$, confirman la centralidad del tema de la justicia predictiva o, más correctamente, de la previsibilidad, celeridad y eficiencia de las decisiones ${ }^{160}$. En el escenario, tal como se mencionó, permanecen las cuestiones relativas, por un lado, a la actividad jurisdiccional y, por otro, a la exigencia de seguridad jurídica ${ }^{161}$.

Aunque de hecho pareciera imposible que la predictividad se le asignara a un juez robot ${ }^{162}$, reviste gran interés la discusión aquí planteada sobre la posibilidad de mejorar la previsibilidad de las decisiones en la óptica de una jurisprudencia más uniforme $y$, por consiguiente, de una mayor seguridad jurídica ${ }^{163}$, gracias al auxilio de los robots y de los algoritmos ${ }^{164}$.

En el actual entorno jurídico y social, cada vez más se le pide al juez que configure las técnicas de protección apropiadas para la implementación efectiva de los intereses representados en el proceso, identificados a través de una reconstrucción

157 Ex multis, ZAGReBelsky, V., "Dalla varietà della giurisprudenza alla unità della giurisprudenza", Cass.pen., 1988, 1576 ss.; Gorla, G., s.v. "Precedente giudiziario", Enc. Giur. Treccani, vol. xxxvI, Roma, 1991; Mattei, U., "Precedente giudiziario e stare decisis", Dig. Disc. Priv., Sez. civile, vol. XIV, Torino, Utet, 1996; TARuffo, M., Precedente e giurisprudenza, cit., 712 ss.; CAdopPI, A., Il valore del precedente nel diritto penale, Torino, Giappichelli, 2007.

158 Véase Dalfino, D., "Giurisprudenza 'creativa' e prevedibilità del 'diritto giurisprudenziale'”, Giusto processo civ., 2017, 1023 ss. En el sentido de que la jurisprudencia, precisamente por su necesaria actividad de interpretación y aplicación de la ley, representa "factor del derecho". Véase también Gorla, G., Diritto comparato e diritto comune europeo, Milano, Giuffrè, 1981, 263 ss. Para diferenciar pragmáticamente la creatividad, destinada a la producción de normas por parte del juez, y la creatividad que consiste semánticamente en la actividad de interpretación del derecho positivo por parte del juez, véase PARDOLESI, R. y PinO, G., "Post-diritto e giudice legislatore. Sulla creatività della giurisprudenza", Foro it., 2017, v, c. 113 ss.

159 Véase Converso, M., “Giudici, 'computer', sentenze”, Foro it., 1990, i, 693 ss.

160 Véase Di Cıоммо, F., "Sulla giustizia ingiusta (dalla giurisprudenza normativa alla giustizia del caso concreto): la vicenda emblematica delle nullità negoziali”, Foro it., 2018, V, 249 ss.

161 Véase Lombardi Vallauri, L., "Verso un sistema esperto giuridico integrale”, Jus, 1996, 157 ss.

162 Véase Alvazzi Del Frate, P., "Interpretazione giudiziale e illuminismo da Beccaria al Code civil", en Rossi, G. y Zanuso, F. (eds.), Attualità e storicità del "Dei delitti e delle pene" a duecentocinquanta anni dalla pubblicazione, Napoli, ESI, 2015, $75 \mathrm{~s}$.

163 Véase LaWlor, R. C., "What computers can do: analysis and prediction of judicial decisions", American Bar Association Journal, 1963.

164 Véase SENOR, M., "Gli algoritmi predittivi nell'amministrazione della giustizia”, La voce dell'agorà, n. $27,2017$. 
general del sistema, y que prosiga seleccionando el remedio que está autorizado a seleccionar ${ }^{165}$. Dado que resolver estos problemas a través de la decisión elaborada por un algoritmo parece una inadecuada simplificación, se propone evitar la idea de la IA como respuesta a la lentitud y la opacidad del sistema de justicia ${ }^{166}$.

Viceversa, la IA puede ser una herramienta útil para conocer y gestionar las experiencias y las aplicaciones que los juristas ya han puesto en marcha en los ámbitos de interés ${ }^{167}$.

Además, el potencial derivado del uso de algoritmos predictivos debe aprovecharse aún más con el fin de garantizar el acceso a la justicia para todos ${ }^{168}$.

Por lo tanto, el problema ya no atañe a una innovación legislativa o a una revolución científica, sino a un enfoque cultural al tema de la justicia humana, ontológicamente diversa de la matemática, de la artificial y, por supuesto, de la "divina".

Alan Turing observó con precisión que "si se espera que una máquina sea infalible, entonces no puede ser también inteligente" ${ }^{169}$.

\section{Referencias}

AA.vv., Calcolabilità giuridica, CARleo, A. (ed.), Bologna, Il Mulino, 2017.

AA.vV., Il vincolo giudiziale del passato. I precedenti, CARLEO, A. (ed.), Bologna, Il Mulino, 2018.

AA.vv., Intelligenza meccanica, Torino, Bollati Boringhieri, 1994.

Aletras, N. et al., "Predicting judicial decisions of the European Court of Human Rights: a natural language processing perspective", Peer Journal of Computer Science, 2:e93, 24 de octubre, 2016.

Ancona, M., "Industria 4.0 e sistema giustizia", Caos. Rivista quadrimestrale per l'innovazione sociale, fasc. 2, 2017.

Ash, E. et al., Judge Writing Style Predicts Decision Tendencies in U.S. Bankruptcy Courts, Technical Report, 2018.

165 Nuzzo, M., "Il problema della prevedibilità delle decisioni: calcolo giuridico secondo i precedenti", en AA.vv., Calcolabilità giuridica, cit., 142. Sobre dichas cuestiones; además, CARTABIA, M., "“Nuovi diritti' e leggi imperfette", Iustitia, n. ${ }^{\circ}$ 2, 2016, 153 ss.

166 Viola, L., Interpretazione della legge con modelli matematici, cit., 26 ss.

167 Вісні, R., "Intelligenza artificiale e diritto", cit., 1777.

168 Véase la Agenda 2030 (onU), objetivo 16.

169 Turing, A. M., "Lecture to the London Mathematical Society on 20 February 1947", ahora en Intelligenza meccanica, cit., 87. 
Asimov, I., Io, Robot, Milano, Bompiani, 1985.

AvitabiLe, L., “Il diritto davanti all'algoritmo", Riv. it. sc. giur., 2017, 319 ss.

Baldwin, R., The Globotics Upheaval. Globalization, Robotics, and the Future of Work, London, Weidenfeld \& Nicolson, 2019.

BaLkin, J. M., “The path of robotics law”, Calif. L. Rev., 2015, 45 ss.

Barocas, S. y Selbst, A. D., "Big data's disparate impact”, Cal. L. Rev., 2016, 104, $671 \mathrm{~s}$.

BARrio Andrés, M. (dir.), Derecho de los robots, Madrid, La Ley, 2018.

Battelli, E., "Il paternalismo giuridico libertario nella prospettiva dell'autonomia privata tra vincoli strutturali e limiti funzionali”, Pol. diritto, 2018, 4, 579 ss.

Belloso Martin, N., "Algoritmos predictivos al servicio de la justicia: una forma de minimizer el riesgo y la incertidumbre", Revista da Faculdade Mineira de Direito, puc Minas / Dossiê - Jorge Eduardo Douglas Price e Raffaele De Giorgi, 2019, 22, 43, 1-31.

BERGÉ, J.-S. et al., "The 'Datasphere'. Data flows beyond control, and the challenges for law and governance", Eur. J. Comp. Law \& Gov., 5, 2, 2018, 144 ss.

BICHI, R., "Intelligenza artificiale e diritto. Intelligenza artificiale tra 'calcolabilità' del diritto e tutela dei diritti", Giur. It., 2019, 7, 1773 ss.

Borgesius, F. Z. (ed.), Discrimination, Artificial Intelligence, and Algorithmic Decision-Making, Strasbourg, Council of Europe, 2018.

Borruso, R., L’informatica nel diritto, Milano, Giuffrè, 2004, 316 s.

CADOPPI, A., Il valore del precedente nel diritto penale, Torino, Giappichelli, 2007.

CAfagGi, F., "Regolazione transnazionale e trasformazioni dello Stato: un'introduzione", en ID. (ed.), Regolazione transnazionale e trasformazioni dello Stato, Bologna, Il Mulino, 2016.

CARdon, D., "Le pouvoir des algorithmes", Pouvoirs, 164, 1, 2018, 63 ss. 
Casonato, C., "Intelligenza artificiale e diritto costituzionale: prime considerazioni”, Dir. pubbl. comp. eur., n. ${ }^{\circ}$ especial, 2019, 101 ss.

Castelli, C. y Piana, D., "Giustizia predittiva. La qualità della giustizia in due tempi”, Questione Giustizia, 4, 2018, 154 ss.

Castronovo, C., Eclissi del diritto civile, Milano, Giuffrè, 2015.

Chander, A., “The racist algorithm?”, Michigan L. Rev., 115, 6, 2017, 1023 ss.

Chen, D. L. y EAGEL, J., "Can machine learning help predict the outcome of asylum adjudications?", en Proceedings of the Association for Computing Machinery Conference on Artificial Intelligence and the Law, 2016.

Clarke, A. C., 2001. A Space Odissey, trad. ital., 2001. Odissea nello spazio, B. Oddera (trad.), Milano, Longanesi, 1972.

Converso, M., “Giudici, ‘computer', sentenze”, Foro it., 1990, I, 693 ss.

Costantino, G., "Il principio di affidamento tra fluidità delle regole e certezza del diritto (Relazione all'Incontro sul tema: 'Norme processuali e mutamenti di giurisprudenza', Roma, 24 maggio 2011)”, Riv. dir. proc., 2011, 5, 1073 ss.

Craglia, M. (ed.), Artificial Intelligence. A European Perspective, Luxembourg, 2018.

CRISCI, S., "Intelligenza artificiale ed etica dell'algoritmo", Foro Amministrativo, $10,1787 \mathrm{~s}$.

DALFINO, D., "Creatività e creazionismo, prevedibilità e predittività", Foro it., 2018, pt. V, c. 385 ss.

DALFino, D., “Giurisprudenza 'creativa' e prevedibilità del 'diritto giurisprudenziale”, Giusto processo civ., 2017, 1023 ss.

De Condorcet, M., Essai sur application de l'analyse à la probabilité des décisions rendues à la pluralité des voix, Paris, 1785.

De FinetTi, B., Teoria delle probabilità. Sintesi introduttiva con appendice critica, Torino, Einaudi, 1970. 
De la Oliva Santos, A., “'Giustizia predittiva', interpretazione matematica delle norme, sentenze robotiche e la vecchia storia del 'Justizklavier'”, Riv. trim. dir. proc. civ., 3, 2019, 883 ss.

De la Oliva Santos, A., Informática, interpretación (¿inexistente por innecesaria o matemática?), De la ley y la vieja historia del “Justizklavier”, Conferencia internacional de estudios "Giustizia predittiva e prevedibilità delle decisioni. Dalla certezza del diritto alla certezza dell'algoritmo?", Università degli studi di Bari Aldo Moro, 5 de octubre de 2018.

De Miguel Beriaín, Í., "Does the use of risk assessments in sentences respect the right to due process? A critical analysis of the Wisconsin v. Loomis ruling", Law Probability and Risk, 17, 1, 2018, 45-53.

De Santis, A. D., s.v. "Processo telematico", en Enc. Giuridica Treccani, Roma, 2015.

Desai, D. R. y Kroll, J. A., "Trust but verify: a guide to algorithms and the law", Harvard J. Law \& Tech., 1, 2018, 35 ss.

Di Cıоммо, F., "Sulla giustizia ingiusta (dalla giurisprudenza normativa alla giustizia del caso concreto): la vicenda emblematica delle nullità negoziali", Foro it ., 2018, v, 249 ss.

Di SABAto, D., "Gli smart contracts: robot che gestiscono il rischio contrattuale", Contr. Impr., 2017, 378 ss.

Dondero, B., "La justice prédictive”, [en línea], disponible en: https://brunodondero.com/2017/02/10/la-justice-predictive, 2017.

Douglas Price, J. E., La decisión judicial, Buenos Aires, Culzoni, 2012.

DuclercQ, J.-B., "Le droit public à l'ère des algorithmes", Rev. Dr. Pub., 5, 2017, 1401 ss.

Fabiani, E., Clausole generali e sindacato della Cassazione, Torino, utet, 2003, 712 ss.

Ferragina, P. y Luccio, F., Il pensiero computazionale. Dagli algoritmi al coding, Bologna, Il Mulino, 2017.

Ferrarese, M. R., Diritto sconfinato, Roma-Bari, Laterza, 2006. 
Freeman, K., "Algorithmic injustice: how the Wisconsin Supreme Court failed to protect due process rights in State v. Loomis", North Caroline Journal of Law and Technology (NCJL \& Tech.), 18, 2016, 75 ss.

Gadamer, H. G., Wahrheit und Methode, Tübingen, trad. ital., Verità e metodo, G. Vattimo (trad.), Milano, Bompiani, 1972, 1990.

GARAPON, A., "Les enjeux de la justice prédictive", Le semaine juridique, éd. générale, Hebdomadaire, n..$^{\circ}$ 1-2, 2017.

Gaudemet, P. Y., "La justice à l'heure des algorithmes”, Rev.Dr.Pub., 3, 2018, 651 ss.

Gentili, A., Senso e consenso. vols. I y II, Storia e teoria, Torino, Giappichelli, 2015.

GIUFFrè, V., Il diritto dei privati nell'esperienza romana. I principali gangli, Napoli, Jovene, 2002.

Gorla, G., s.v. "Precedente giudiziario”, en Enc. Giur. Treccani, xxxvi, Roma, 1991.

Gorla, G, Diritto comparato e diritto comune europeo, Milano, Giuffrè, 1981.

Grossi, P., Ritorno al diritto, Roma-Bari, Laterza, 2015.

HARris, A., "Judicial decision making and computers", Vill. L. Rev. 12, 272, 1967, $312 \mathrm{~s}$.

Hoffmann-Riem, W., "Verhaltenssteuerung durch Algorithmen. Eine Herausforderung für das Recht”, Archiv des öffent. Rechts, 142, 2017, 2 ss.

Holder, C. et al., "Robotics and law: key legal and regulatory implications of the robotic age", I, Computer Law and Security Review, 2016, 384 ss.

Holmes, O. W., "The path of the law”, Harvard Law Review, 457, 1897 ss.

IASELLI, M., "È ipotizzabile l'avvocato-robot o il giudice-robot? Le applicazioni dell'intelligenza artificiale nel settore legale”, Altalex.com, 15 de junio, 2017.

Irti, N. y Severino, E., "Le domande del giurista e le risposte del filosofo (un dialogo tra diritto e tecnica)", en Contratto e Impresa, 2006, 665 ss.

IRTI, N., "Calcolabilità weberiana e crisi della fattispecie”, Riv. dir. civ., 2014, 987 ss. 
IRTI, N., "Capitalismo e calcolabilità giuridica (letture e riflessioni)", Riv. Società, 2015,801 ss.

IRTI, N., "La crisi della fattispecie", Riv.dir. proc., 2014, 43 ss.

IRTI, N., "Per un dialogo sulla calcolabilità giuridica", Riv. dir. proc., 2016, 921 ss.

IRTI, N., Un diritto incalcolabile, Torino, Giappichelli, 2016.

KAPLAN, J., Intelligenza artificiale. Guida al futuro prossimo, Roma, LUISS University Press, 2017, 52 ss.

KaPlan, R., The Nothing that is. A Natural History of Zero, trad. ital., Zero. Storia di una cifra, S. Galli y C. Capararo (trads.), Milano, RCs, 2014.

Katsh, E. y Rabinovich-Einy, O., Digital Justice. Technology and the Internet of Disputes, Oxford, Oxford University Press, 2017.

Keats Citron, D. y Pasquale, F., "The scored society: due process for automated predictions", Washington Law Review, 89, 1, 2014, 1 ss.

Kelsen, H., Society and Nature. A Sociological Inquiry, Chicago (Ill.), The University of Chicago Press, 1943, trad. ital., Società e natura. Ricerca sociologica, Torino, Einaudi, 1953, 18 ss.

Kroll, J. A., "The fallacy of inscrutability”, Phil. Trans. R. Soc., 376, 2133, 2018.

LAmON, B., "La profession d'avocat et la justice prédictive: un bel outil pour le développement du droit", Récueil Dalloz, 14, 2017.

LAWLOR, R. C., "What computers can do: analysis and prediction of judicial decisions", American Bar Association Journal, 49, 4, 1963, 337 ss.

Leibniz, G. W., "Princìpi ed esempi della scienza generale", en Scritti di logica, BARONE, F. (ed.), Milano, Mondadori, 2009.

Lin, P. et al., Robot Ethics, MIT Press, 2012.

Lin, P. et al., "Robot ethics: mapping the issues for a mechanized world", Artificial Intelligence 175, 2011, 944 ss.

LIPARI, N., "I civilisti e la certezza del diritto", Riv. dir. civ., 2015, 1115 ss. 
Lombardi Vallauri, L., "Verso un sistema esperto giuridico integrale", Jus, 1996, 157 ss.

LuCiani, M., "Garanzie ed efficienza nella tutela giurisdizionale”, Dir. soc., 2014, 433 ss.

LuCIANI, M., "La decisione giudiziaria robotica", Riv. Associaz. Italiana Costituzionalisti, 2018, 3, 872 ss.

Luminoso, A. (ed.), Diritto e crisi, Milano, Giuffrè, 2016.

Manes, V., Il giudice nel labirinto, Profili di intersezioni tra diritto penale e fonti sovranazionali, Roma, Dikegiuridica, 2012.

Marcoz, G., "Intelligenza artificiale e giustizia, perché è importante la Carta etica europea", Agenda digitale.eu, 15 de febrero, 2019.

Martinay, A. y Mazens, M., "Regards sur les 'promesses' de la justice predictive”, [en línea], disponible en: http://blogs.univ-poitiers.fr/c-marcon

Martínez García, I., "Inteligencia y derechos humanos en la sociedad digital", Cuadernos Electrónicos de Filosofía del Derecho, 2019, 40, 168-189.

Mattei, U., "Precedente giudiziario e "stare decisis"”, Dig. Disc. Priv., Sez. civile, vol. XIV, Turín, UTET, 1996.

MAtTERA, R., “Decisione negoziale e giudiziale: quale spazio per la robotica?”, Nuova giur. civ. comm., 2019, 201 ss.

Meккі, M., "If code is law, then code is justice? Droit et algorythmes", Gazette $d u$ Palais, 27 de junio, 2017.

Mengoni, L., "Spunti per una teoria delle clausole generali”, Riv. critica dir. privato, 1986, 5 ss.

Morelli, C., "Algoritmi e diritti umani: si rischia la collisione (immaginate a danno di chi?)", Altalex.com, 9 de julio, 2018.

Nazzaro, A. C., "Macchine intelligenti ('smart cars'): assicurazione e tutela della 'privacy'. Smart cars insurance and protection of privacy", Diritto del mercato assicurativo e finanziario, 2018, 1, 77 ss. 
Neumann, J. von, The Computer \& the Brain, New Haven, Yale University Press, 1958, trad. ital., Computer e cervello, P. Bartesaghi (trad.), Milano, Il Saggiatore, 2014.

Nieva Fenoll, J., Inteligencia artificial y proceso judicial, Madrid, Marcial Pons, 2018.

Noto La Diega, G., "Against the de-humanisation of decision-making”, JIPITEC, 9, $3,2018$.

O’NeIL, C., Weapons of Math Destruction: How Big Data Increases Inequality and Threatens Democracy, London, Penguin, 2016.

Olhede, S. C. y Wolfe, P. J., "The growing ubiquity of algorithms in society: implications, impacts, and innovations", Phil. Trans. R. Soc., 2018, 1 ss.

Pacchioni, G., "I poteri creativi della giurisprudenza", Riv. dir. comm., 1912, 41 ss.

PALMERIN E. et al., "RoboLaw: towards a European framework for robotics regulation”, Robotics and Autonomous Systems, 2016, 78 ss.

Pardolesi, R. y Pino, G., "Post-diritto e giudice legislatore. Sulla creatività della giurisprudenza", Foro it., 2017, V, c. 113 ss.

Pasquale, F., The Black Box Society: The Secret Algorithms that Control Money and Information, Cambridge-London, Harvard University Press, 2015.

Passanante, L., Il precedente impossibile. Contributo allo studio del diritto giurisprudenziale nel processo civile, Torino, Giappichelli, 2018.

Pauliat, H., "La décision administrative et les algorithmes: une loyauté à consacrer", Rev.dr. pub., 3, 2018, 641 ss.

Pedreschi, D. et al., "Open the black box. Data-driven explanation of black box decision systems", ArXiv, 1, 2018, 1 ss.

PINO, G., "La gerarchia delle fonti del diritto. Costruzione, decostruzione, ricostruzione", Ars interpretandi, xvI, 2011, 53 ss.

Pizzetti, F. (ed.), Intelligenza artificiale, protezione dei dati personali e regolazione, Torino, Giappichelli, 2018. 
Poisson, S. D., Recherches sur la probabilité des jugements en matière criminelle et en matière civile, Paris, Bachelier, 1837.

Poli, G. G., "Il sistema delle fonti del processo civile telematico", Riv. dir. proc., 2016, 4-5, 1201 ss.

Punzi, A., L'ordine giuridico delle macchine. La Mettrie - Helvétius - D'Holbach. L'uomo macchina verso l'intelligenza collettiva, Torino, Giappichelli, 2003.

QuARTA, E., “Giustizia e predizione: l'algoritmo che legge il futuro”, Giustizia insieme, 10 de marzo, 2019.

QuARTA, E., “L'algoritmo giuridico che realizza l'antico sogno dell'uomo di visualizzare scenari avveniristici: la giustizia nell'era 4.0", Personaedanno.it, 21 de febrero, 2019.

Resta, G., "Governare l'innovazione tecnologica: decisioni algoritmiche, diritti digitali e principio di uguaglianza”, Pol. diritto, 2, 2019, 199 ss.

Rodotà, S., Tecnologie e diritti, Bologna, Il Mulino, 1995.

Romano, B., Dalla metropoli verso internet. Persona. Libertà. Diritto, Torino, Giappichelli, 2017.

Rossi, G. y Zanuso, F. (eds.), Attualità e storicità del "Dei delitti e delle pene" a duecentocinquanta anni dalla pubblicazione, Napoli, ESI, 2015.

Ruffolo, U., Intelligenza artificiale e responsabilità, Milano, Giuffrè, 2018.

Rulli, E., "Giustizia predittiva, intelligenza artificiale e modelli probabilistici. Chi ha paura degli algoritmi?”, Analisi Giuridica dell'Economia, 2018, 533 ss.

SARtor, G., Artificial Intelligence: Challenges for EU Citizens and Consumers, PE 631.043, Brussels, January 2019.

SARtor, G., Intelligenza artificiale e diritto. Un'introduzione, Milano, Giuffrè, 1996.

SASSI, S., "Gli algoritmi nelle decisioni pubbliche tra trasparenza e responsabilità", Analisi Giuridica dell'Economia, 2019, 1, 109 ss.

SAUvÉ, J.-M., Le juge administratif et l'intelligence artificielle, Conférence des présidents des jurisdictions administratives, Rennes, 20 de abril de 2018. 
SCHERER, M. U., "Regulating artificial intelligence systems: risks, challenge, competences, and strategies", Har. J.L. \& Tech., 29, 2016, 354 ss.

Schiavone, A., "Ius". L'invenzione del diritto in Occidente, Torino, Einaudi, 2005, 5 ss.

SEVERIno, E., La potenza dell'errare, Milano, Rizzoli, 2013, 87 ss.

Sннн, H., “Algorithmic accountability”, Phil. Trans. R. Soc., 376, 2128, 2018.

Simoncini, A. y Suweis, S., "Il cambio di paradigma nell'intelligenza artificiale e il suo impatto sul diritto costituzionale", Riv. filosofia dir., 1, 2019, 87 ss.

Simoncini, A., "L'algoritmo incostituzionale: intelligenza artificiale e il futuro delle libertà”, BioLaw Journal - Rivista di BioDiritto, 2019, 1, 63 s.

Solar CAyón, J. I., La inteligencia artificial jurídica. El impacto de la innovación tecnológica en la práctica del derecho y el mercado de servicios jurídicos, Cizur Menor, Aranzadi, 2019.

Susskind, R., Tomorrow's Lawyers. An Introduction to Your Future, 2. a ed., Oxford, Oxford University Press, 2017.

Taddei Elmi, G., Corso di informatica giuridica, Napoli, Simone, 2007.

TARUfFo, M., "Legalità e giustificazione della creazione giudiziaria del diritto", Riv. trim. dir. e proc. civ., 2001, 11 ss.

TARuffo, M., "Precedente e giurisprudenza", Riv. trim. dir. e proc. civ., 2007, 710 ss.

Tegmark, M., Vita 3.0. Essere umani nell'era dell'intelligenza artificiale, Milano, Raffaello Cortina, 2018.

Temme, M., "Algorithms and transparency in view of the new General Data Protection Regulation", Eur. Data Prot. L. Rev., 3, 4, 2017, p. 473 ss.

Thaler, R. y Sunstein, C. R., Nudge. La spinta gentile. La nuova strategia per migliorare le nostre decisioni su denaro, salute, felicità, Milano, Feltrinelli, 2018.

TisCornia, D., Il diritto nei modelli dell'intelligenza artificiale, Bologna, CluEB, 1996. 
Vettori, G., "Effettività delle tutele (dir. civile)", Enciclopedia del diritto, Milano, Giuffrè, 2017.

Viola, F. et al., Le ragioni del diritto, Bologna, Il Mulino, 2017.

Viola, L., "Giustizia predittiva”, www.treccani.it, 2018.

Viola L., Interpretazione della legge con modelli matematici. Processo, a.d.r., giustizia predittiva, 2. ${ }^{\text {a }}$ ed., Milano, Centro Studi Diritto Avanzato, 2018.

Viola L., "L'interpretazione della legge ex art. 12 Preleggi si basa su un algoritmo", Giustiziacivile.com, 22 de febrero, 2019.

Weber, M., Economia e società, Milano, Bompiani, 1947.

ZACCARIA, G., Ermeneutica e giurisprudenza. I fondamenti filosofici nella teoria di Hans Georg Gadamer, Milano, Giuffrè, 1984.

ZAGREBELSKY, V., "Dalla varietà della giurisprudenza alla unità della giurisprudenza”, Cass. pen., 1988, 1576 ss.

ZelLini, P., La dittatura del calcolo, Milano, Adelphi, 2018. 